\title{
Duality between the Deconfined Quantum-Critical Point and the Bosonic Topological Transition
}

\author{
Yan Qi Qin, ${ }^{1}$ Yuan-Yao He, ${ }^{2}$ Yi-Zhuang You, ${ }^{3}$ Zhong-Yi Lu, ${ }^{2}$ Arnab Sen, ${ }^{4}$ \\ Anders W. Sandvik, ${ }^{5}$ Cenke $\mathrm{Xu},{ }^{6}$ and $\mathrm{Zi}$ Yang Meng ${ }^{1}$ \\ ${ }^{1}$ Institute of Physics, Chinese Academy of Sciences, Beijing 100190, China \\ and University of Chinese Academy of Sciences, Beijing 100049, China \\ ${ }^{2}$ Department of Physics, Renmin University of China, Beijing 100872, China \\ ${ }^{3}$ Department of Physics, Harvard University, Cambridge, Massachusetts 02138, USA \\ ${ }^{4}$ Department of Theoretical Physics, Indian Association for the Cultivation of Science, \\ Jadavpur, Kolkata 700032, India \\ ${ }^{5}$ Department of Physics, Boston University, 590 Commonwealth Avenue, \\ Boston, Massachusetts 02215, USA \\ ${ }^{6}$ Department of Physics, University of California, Santa Barbara, California 93106, USA
}

(Received 3 June 2017; revised manuscript received 14 August 2017; published 22 September 2017)

Recently, significant progress has been made in $(2+1)$-dimensional conformal field theories without supersymmetry. In particular, it was realized that different Lagrangians may be related by hidden dualities; i.e., seemingly different field theories may actually be identical in the infrared limit. Among all the proposed dualities, one has attracted particular interest in the field of strongly correlated quantum-matter systems: the one relating the easy-plane noncompact $\mathrm{CP}^{1}$ model $\left(\mathrm{NCCP}^{1}\right)$ and noncompact quantum electrodynamics (QED) with two flavors $(N=2)$ of massless two-component Dirac fermions. The easyplane $\mathrm{NCCP}^{1}$ model is the field theory of the putative deconfined quantum-critical point separating a planar $(X Y)$ antiferromagnet and a dimerized (valence-bond solid) ground state, while $N=2$ noncompact QED is the theory for the transition between a bosonic symmetry-protected topological phase and a trivial Mott insulator. In this work, we present strong numerical support for the proposed duality. We realize the $N=2$ noncompact QED at a critical point of an interacting fermion model on the bilayer honeycomb lattice and study it using determinant quantum Monte Carlo (QMC) simulations. Using stochastic series expansion QMC simulations, we study a planar version of the $S=1 / 2 \mathrm{~J}-Q$ spin Hamiltonian (a quantum $X Y$ model with additional multispin couplings) and show that it hosts a continuous transition between the $X Y$ magnet and the valence-bond solid. The duality between the two systems, following from a mapping of their phase diagrams extending from their respective critical points, is supported by the good agreement between the critical exponents according to the proposed duality relationships. In the $J-Q$ model, we find both continuous and first-order transitions, depending on the degree of planar anisotropy, with deconfined quantum criticality surviving only up to moderate strengths of the anisotropy. This explains previous claims of no deconfined quantum criticality in planar two-component spin models, which were in the stronganisotropy regime, and opens doors to further investigations of the global phase diagram of systems hosting deconfined quantum-critical points.

DOI: 10.1103/PhysRevX.7.031052

\author{
Subject Areas: Computational Physics, \\ Condensed Matter Physics, \\ Statistical Physics
}

\section{INTRODUCTION}

A duality in physics is an equivalence of different mathematical descriptions of a system or a state of matter, established through a mapping by change of variables. In classical statistical mechanics, the most famous duality is the Kramers-Wannier duality of the two-dimensional Ising

Published by the American Physical Society under the terms of the Creative Commons Attribution 4.0 International license. Further distribution of this work must maintain attribution to the author(s) and the published article's title, journal citation, and DOI. model [1]. Here, the low- and high-temperature expansions of the partition function can be related to each other by identifying a one-to-one correspondence between the terms in the two different series, thus establishing an exact mapping between the ordered and disordered phases and the corresponding collective variables. In this case, the critical point is also a self-duality point. In the 3D Ising model, one can instead find a different model whose hightemperature expansion stands in a direct one-to-one correspondence with the low-temperature expansion of the Ising gauge model [2,3]. Many other examples of dualities have been established, e.g., the well-known equivalence between the 3D $O(2)$ Wilson-Fisher fixed 
point and the 3D Higgs transition with a noncompact $U(1)$ gauge field [4-7].

In analogy with the Ising examples mentioned above, it is sometimes possible to transform a quantum field theory at strong coupling into an equivalent dual theory at weak coupling. The untractable original problem can then be solved by means of perturbative methods applied to the dual theory. Such strong-weak duality (and the more general " $S$ duality" form) was established in certain supersymmetric Yang-Mills theories [8-11] and Abelian gauge theory without supersymmetry [12-14]. In 1D quantum systems (i.e., in $1+1$ space-time dimensions) a wellknown fermion-boson duality is achieved by bosonization of an interacting fermion system through a nonlocal transformation [15-19]. Usually, in the bosonized formalism interactions can be more easily treated than in the original fermion model.

In cases where no formal mapping is known, two Lagrangians that look different in the ultraviolet may still flow (under the renormalization group) to the same theory in the infrared; i.e., these seemingly different field theories actually represent exactly the same low-energy physics. Such a duality goes a step beyond the more familiar concept of universality, by which systems (models or real materials) with the same dimensionality and global symmetries exhibit identical scaling properties at their classical or quantum-critical points. Such systems share the same effective critical low-energy field-theory description. For example, the critical points of the Bose-Hubbard model and the quantum rotor model are in the same universality class. A duality transformation usually changes the description of the system into a form based on nonlocal objects or defects of the original description. On a practical level, the existence of a dual field theory means that there is a nontrivial choice of which description to use, and one of them (and not necessarily the originally most obvious one) may pose a more tractable setup for calculations.

Even if no strong-weak transformation exists (or is known), a difficult or nontractable strong-coupling problem can sometimes be shown to be dual to a different strongcoupling problem that is tractable with some specific computational technique. In particular, the dual problem may be more easily solvable using powerful numerical (lattice) methods. This numerical amenability aspect of dualities has so far not been emphasized in the literature, but it is apparent in the work we present here. We explore a recently proposed duality between two different strongly coupled $(2+1)$ D Lagrangians that respectively involve fermionic and bosonic matter fields coupled with a gauge field [20-22]. Both theories are of great current interest in the context of strongly correlated electrons in two dimensions. Our aim here is to identify a duality between the systems by establishing corresponding lattice models realizing the two low-energy theories, and one of them, the bosonic one, has substantially superior scaling properties of the computational effort (as a function of the system size) in quantum Monte Carlo (QMC) simulations.

We follow the recent proposal that $(2+1) \mathrm{D}$ quantum electrodynamics (QED) with noncompact gauge field and two flavors of Dirac fermions is dual to the critical point of the easy-plane $\mathrm{NCCP}^{1}$ model (the bosonic QED with two flavors of complex bosons) [20-22]. On the lattice, we realize the former with an interacting fermion model with spin-orbit coupling on the bilayer honeycomb $(\mathrm{BH})$ lattice, which hosts a quantum phase transition between a (bosonic) symmetry-protected topological state and a trivial Mott insulator [23-25]. It was proposed that this transition is described by $N=2$ noncompact QED [26,27]. To realize the low-energy physics of the $\mathrm{NCCP}^{1}$ theory, in this paper we introduce a planar variant of the spin $S=1 / 2 \mathrm{~J}-Q$ Hamiltonian (a Heisenberg model with additional multispin couplings [28]), dubbed the easy-plane $J-Q$ (EPJQ) model, and show that it hosts a deconfined quantum-critical point [29-31] separating antiferromagnetic (AFM) and dimerized [valence-bond-solid (VBS)] ground states (similar to the case of the $J-Q$ model with full spin $\mathrm{SU}(2)$ symmetry [32], but with different universality due to the lowered symmetry). Our QMC results establish the critical-point universality and duality between the phase diagrams of the two models. With the EPJQ model being much easier to study on large scales with QMC simulations than the fermionic model, the duality that we establish here allows for detailed studies of the topological transition of the latter, through the analogue of the deconfined quantum-critical point. The phase diagrams and dualities we study are illustrated in Fig. 1.

We summarize our main results and insights as follows.

(1) The EPJQ spin model hosts both deconfined quantum criticality and a first-order transition between the easy-plane AFM and VBS states, depending on a single anisotropy parameter. The existence of deconfined quantum criticality in planar two-component spin models had been seriously questioned in several works over the years [35-38]. Most recently it was argued [38], within a $4-\epsilon$ expansion of the $\mathrm{CP}^{N-1}$ field theory, that a tricritical point separating continuous (for weak anisotropy) and first-order (for strong anisotropy) phase transitions between the AFM and VBS phases in Fig. 1 may exist, but only for large $N$ (and the transitions for smaller $N$ are always first order). In our model, we find that continuous transitions are obtained only up to moderate values of the anisotropy, thus showing that the tricritical point extends all the way down to the physically most interesting case of $N=2$. The previous works were all carried out with strongly anisotropic models and were in the first-order regime.

(2) Within small statistical error bars, our simulations of the EPJQ and BH models verify the key quantitative predictions based on the dualities proposed in 
(a) $\mathrm{BH}$ model

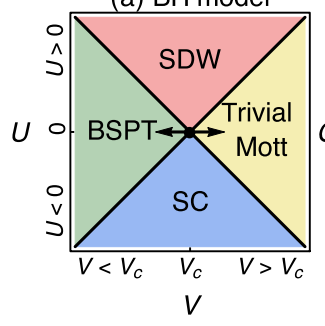

(b) EPJQ model

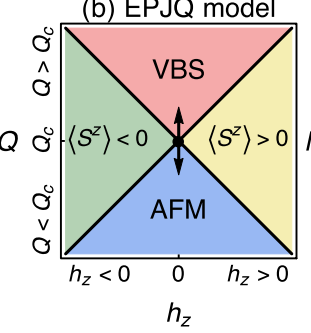

(c) $N=2$ QED

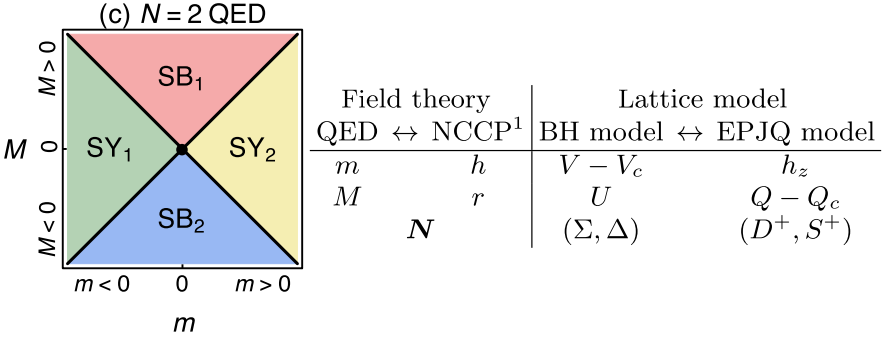

FIG. 1. Schematic phase diagrams of (a) the bilayer honeycomb (BH) model, (b) the easy-plane $J-Q_{3}$ (EPJQ) model, and (c) the $N=2$ QED theory. In (a), the BH model contains two symmetry-breaking phases, the spin-density wave (SDW) and superconducting (SC) phases, and two symmetric phases, the bosonic symmetry-protected topological (BSPT) and the trivial Mott-insulating phases. In (b), the EPJQ model also contains two symmetry-breaking phases, the Néel antiferromagnetic (AFM) phase and the valence-bond solid (VBS) phase, and two spin-polarized phases induced by an external staggered field. In (c), as was shown in Refs. [22,27,33], when tuning the two masses $m$ and $M$, the $N=2$ QED theory also has two symmetry-breaking (SB) phases and two symmetric (SY) phases, one of which is the BSPT state. In all models, the four phases merge at the deconfined quantum-critical point. Phases of the same color can be mapped to each other among the models, following the duality relations proposed in the table on the right-hand side. The double arrows in (a) and (b) indicate the quantum phase transitions investigated numerically in this paper, with the paths chosen to cross the respective critical points in the physically most interesting and computationally most tractable way. In (c), recent lattice QED calculations have focused exclusively on the critical point separating all the phases [34].

Ref. [22]. These dualities had been analyzed from many perspectives [22] but were discussed at a purely theoretical level and demand either numerical or experimental confirmation. Our work provides a quantitative verification, strongly supporting the dualities by confirming some of their most remarkable nontrivial consequences, and thus also implicitly supporting the more elementary dualities [39,40] upon which the derivations in Ref. [20-22] were based.

(3) Building on the tricritical point we identify here within the EPJQ spin model, our work suggests other directions in which to expand the web of dualities, by looking for the analogous point within extensions of the fermionic field theory and the BH model.

The paper is organized as follows. In Sec. II, we present the details of the two field theories and their putative duality, and in Sec. III, we define the lattice models and the proposed mappings relating their phase diagrams to each other. In Secs. IV and V, we present the numerical results for the EPJQ and BH models, respectively. We conclude with a brief summary and discussion of the results in Sec. VI. In Appendix A, we present further technical details on the analysis of the critical exponents, and in Appendix B, we compare results for different variants of the EPJQ model (with different degrees of spin anisotropy).

\section{CONTINUUM FIELD THEORIES}

The bosonic particle-vortex duality we mention in the Introduction was recently generalized to a model with fermionic matter [40-48], in the form of a $(2+1)$ D QED Lagrangian with a single flavor of a two-component Dirac fermion and noncompact gauge field, i.e., $N=1$ QED.
This theory is dual to that of a noninteracting Dirac fermion in the infrared limit [49]. Based on this $N=1$ duality, Ref. [50] showed that $(2+1) \mathrm{D}$ QED with noncompact gauge field and $N=2$ flavors of Dirac fermions is selfdual. This is also a fermionic version of the self-duality of the easy-plane $\mathrm{NCCP}^{1}$ model (which can be regarded as $N=2$ bosonic QED) [29,30,51]. The self-duality of the $N=2$ QED Lagrangian was also verified with different derivations $[20,48,52]$. Unlike the case of $N=1$, there is no equivalent noninteracting description of $(2+1) \mathrm{D}$ QED with $N=2$, however. Because of its self-duality, $N=2$ QED hosts an (emergent) $O(4)$ symmetry in the infrared, which factorizes into the two independent $\mathrm{SU}(2)$ flavor symmetries on the two sides of the self-dual point.

More recently, based on the previous fermion-boson duality $[39,40]$, it was argued that $N=2$ QED is also dual to the easy-plane $\mathrm{NCCP}^{1}$ model at the critical point [20-22]. These two field theories can be written as

$$
\begin{aligned}
\mathcal{L}_{\mathrm{QED}} & =\bar{\psi} \gamma(\partial-i a) \psi+m \bar{\psi} \psi+M \bar{\psi} \sigma^{3} \psi, \\
\mathcal{L}_{\mathrm{CP}}^{1} & =|(\partial-i b) z|^{2}+g|z|^{4}+r z^{\dagger} z+h z^{\dagger} \sigma^{3} z,
\end{aligned}
$$

where $\psi$ and $z$ are two-component Dirac fermion and complex boson fields coupled to noncompact $U(1)$ gauge fields, $a$ and $b$, respectively. The duality maps the variables $(m, M)$ to $(h, r)$. Moreover, both theories in Eq. (1) are individually self-dual. The putative duality between the two theories implies that the easy-plane $\mathrm{NCCP}^{1}$ model should also have an emergent $O(4)$ symmetry at its critical point, which is not immediately obvious in Eq. (1b). The corresponding $O(4)$ order parameter is

$$
\mathbf{N}=\left(z^{\dagger} \sigma^{x} z, z^{\dagger} \sigma^{y} z, \operatorname{Re}\left[\mathcal{M}_{b}\right], \operatorname{Im}\left[\mathcal{M}_{b}\right]\right),
$$


where $\mathcal{M}_{b}$ is the monopole operator (gauge flux annihilation operator) of the gauge field $b$.

The proposed duality between the two theories in Eq. (1) leads to very strong predictions for relationships between their properties. For example, the scaling dimension of $m$ in $N=2$ QED should be precisely the same as the scaling dimension of $h$ in the easy-plane $\mathrm{NCCP}^{1}$ at its critical point $r=0$, while the scaling dimension of $M$ should be the same as that of $r$. Also, as a consequence of these dualities, i.e., the emergent $O(4)$ symmetry of the two theories, the four components of $\mathbf{N}$ should all have the same scaling dimension at the critical point.

Although the duality between Eqs. (1a) and (1b) can be checked and derived based on various arguments [20-22], the predictions still need quantitative proofs, either experimentally or numerically. The numerical "simulation" route is the most direct way to address the mathematical validity aspects of the dualities, and with this issue settled the experiments can better focus on the possibility to realize the low-energy physics described by the theories within specific materials and external conditions.

Both Eqs. (1a) and (1b) are strongly interacting conformal field theories, and there is no obvious analytical method that can provide rigorous results for either case. However, both theories can presumably be realized using lattice models, which can be simulated using numerical methods. The goal of this work is to compare the quantitative properties of such lattice models and look for evidence of the proposed duality. As we show in later sections, within small error bars of the critical exponents obtained using QMC simulations, our results confirm the key predictions of the duality.

Because the duality of the two theories in Eq. (1) was derived based on the assumption of the basic fermionboson duality $[39,40]$, a proof of the former duality indirectly also proves the latter. In principle, this result can lead to a number of further dualities between different fermionic and bosonic Lagrangians. Thus, the impact of our work is not limited to the proof of the duality between Eqs. (1a) and (1b), but also provides justification for many other cases.

\section{LATTICE MODELS}

The easy-plane $\mathrm{NCCP}^{1}$ model is the field theory that presumably describes the deconfined quantum-critical point between an in-plane $(X Y)$ antiferromagnet and a valence-bond solid $[29,30,51]$. This transition in the case of full $\mathrm{SU}(2)$ symmetry of the Hamiltonian has been realized by the $J-Q$ and related models, and these have been extensively simulated numerically using unbiased QMC techniques [28,32,53-62]. Although there are studies that indicate that some version of the $J-Q$ model with an inplane spin symmetry and other $U(1)$ symmetric models should lead to a first-order transition [35-38], in this work we demonstrate that a different model, the EPJQ model, instead leads to a continuous transition in some regions of its parameter space (we also note there is a recent QMC work on the extended Hubbard model of hard-core bosons on the kagome lattice, suggesting a similar continuous easy-plane phase transition [63], consistent with our finding here). The $r$ and $h$ terms in Eq. (1) correspond to the distance from the critical point, $Q-Q_{c}$, and the staggered magnetic field $h_{z}(-)^{i} S_{i}^{z}$, respectively, in the lattice model. The components of the $\mathrm{O}(4)$ vector in Eq. (2) correspond to the two-component easy-plane Néel and two-component VBS (dimer) order parameters of the EPJQ model.

The $N=2$ QED action has been simulated directly using a lattice QED model [34], and the scaling dimension of $M$ is computed in this way. Also, $N=2$ QED with a noncompact $\mathrm{U}(1)$ gauge field is the effective theory that describes the transition between the bosonic symmetryprotected topological (BSPT) state and a trivial Mott state in 2D [26,27]. This transition was also realized in an interacting fermion model on a bilayer honeycomb lattice introduced in Refs. [23,24] and simulated [23-25,64] with a determinantal QMC method (DQMC) $[65,66]$. The $m$ and $M$ terms in Eq. (1) correspond to two different interactions in the lattice model, namely, the interlayer pair hopping $V-V_{c}$, measured with respect to its critical value, and the Hubbard-like on-site interaction $U$; the Hamiltonian is specified in detail below. The lattice model of Ref. [24] has an exact $\mathrm{SO}(4)$ symmetry that precisely corresponds to the proposed emergent symmetry of the $N=2$ QED. It should further be noted that the fermions in the $\mathrm{BH}$ model do not directly correspond to the Dirac fermions of the $N=2$ QED action, because the former are not coupled to any dynamical gauge field. The relation between the two systems instead arises from the correspondence of the gauge-invariant fields of $N=2$ QED to the low-energy bosonic excitations of the $\mathrm{BH}$ model.

Using the BH model and the EPJQ model, the duality between the $N=2$ QED and the $\mathrm{NCCP}^{1}$ field theories can be realized on the lattice. The $O(4)$ vector $\mathbf{N}$ can also be conveniently defined in both lattice models, with explicit forms that we explain below. Thus, the two systems can be investigated and compared via unbiased large-scale QMC simulations - the pursuit and achievement of this work.

In the following, we first define the microscopic lattice models in detail. In the subsequent sections, Secs. IV and V, we present comparative numerical studies of the models and demonstrate strong support for the duality relations listed in the table in Fig. 1.

\section{A. Bilayer honeycomb model}

The BH model is a fermionic model defined on a honeycomb lattice [23-25,64]. On each site, we define four flavors of fermions (two layers $\times$ two spins);

$$
c_{i}=\left(c_{i 1 \uparrow}, c_{i 1 \downarrow}, c_{i 2 \uparrow}, c_{i 2 \downarrow}\right)^{\top} .
$$


The Hamiltonian is

$$
H_{\mathrm{BH}}=H_{\text {band }}+H_{\text {int }},
$$

where the band and interaction terms are given by

$$
\begin{aligned}
H_{\mathrm{band}} & =-t \sum_{\langle i j\rangle} c_{i}^{\dagger} c_{j}+\lambda \sum_{\langle i j\rangle} i \nu_{i j}\left(c_{i}^{\dagger} \sigma^{3} c_{j}+\text { H.c. }\right), \\
H_{\mathrm{int}} & =V \sum_{i}\left(c_{i 1 \uparrow}^{\dagger} c_{i 2 \uparrow} c_{i 1 \downarrow}^{\dagger} c_{i 2 \downarrow}+\text { H.c. }\right)
\end{aligned}
$$

where $\langle i j\rangle$ and $\langle\langle i j\rangle\rangle$ denote nearest-neighbor intralayer site pairs, respectively. The band Hamiltonian $H_{\text {band }}$ is just two copies of the Kane-Mele model [67], which drives the fermion into a quantum spin Hall state with spin Hall conductance $\sigma_{\mathrm{sH}}= \pm 2$ (depending on the sign of the spinorbit coupling $\lambda$ ). Including a weak interaction $V$, the bilayer quantum spin Hall state automatically becomes a BSPT state $[24,25,68]$, where only the bosonic $O(4)$ vector $\mathbf{N}$ remains gapless (and protected) at the edge, while the fermionic excitations are gapped out (as we discuss in more detail below). However, a strong interlayer pair-hopping interaction $V$ eventually favors a direct product state of antibonding Cooper pairs. In the strong interaction limit $(V \rightarrow \infty)$, the ground state of the $\mathrm{BH}$ model reads

$$
|\mathrm{GS}\rangle=\prod_{i}\left(c_{i 1 \uparrow}^{\dagger} c_{i 1 \downarrow}^{\dagger}-c_{i 2 \uparrow}^{\dagger} c_{i 2 \downarrow}^{\dagger}\right)\left|0_{c}\right\rangle
$$

with $\left|0_{c}\right\rangle$ being the fermion vacuum state. This state has no quantum spin Hall conductance, i.e., $\sigma_{\mathrm{sH}}=0$, and, more importantly, it is a direct product of local wave functions, hence dubbed the trivial Mott insulator state. It was found numerically that there is a direct continuous transition between the BSPT and the trivial Mott phases at $V_{c} / t=2.82(1)[24,64]$, where the single-particle excitation gap does not close but the excitation gap associated with the bosonic $O(4)$ vector closes and the quantized spin Hall conductance changes from \pm 2 to 0 .

The low-energy bosonic fluctuations around the critical point form an $O(4)$ vector, with $\mathbf{N}=$ $(\operatorname{Re} \Sigma, \operatorname{Im} \Sigma, \operatorname{Re} \Delta, \operatorname{Im} \Delta)$, and the components are

$$
\begin{aligned}
& \Sigma_{i}=(-1)^{i}\left(c_{i 1 \uparrow}^{\dagger} c_{i 2 \downarrow}+c_{i 2 \uparrow}^{\dagger} c_{i 1 \downarrow}\right), \\
& \Delta_{i}=\left(c_{i 1 \downarrow} c_{i 1 \uparrow}-c_{i 2 \downarrow} c_{i 2 \uparrow}\right),
\end{aligned}
$$

where $\Sigma$ carries spin and $\Delta$ carries charge. The BH model Eq. (4) respects the global $\mathrm{SO}(4)$ symmetry of the vector $\mathbf{N}$. If the symmetry is lowered to $U(1)_{\text {spin }} \times U(1)_{\text {charge }}$, then, based on the analysis of $N=2 \mathrm{QED}$, in principle the mass term $M \bar{\psi} \sigma^{3} \psi$ is allowed; hence, the BSPT-Mott transition is unstable towards spontaneous symmetry breaking of the remaining symmetries. The symmetry of the mass term $M \bar{\psi} \sigma^{3} \psi$ is identical to the following Hubbard-like interaction [both forming a $(1,1)$ representation of the $\mathrm{SO}(4)$ ]:

$$
\frac{U}{2} \sum_{i}\left(\Delta_{i}^{\dagger} \Delta_{i}+\Delta_{i} \Delta_{i}^{\dagger}-\Sigma_{i}^{\dagger} \Sigma_{i}-\Sigma_{i} \Sigma_{i}^{\dagger}\right)=U \sum_{i} \rho_{i \uparrow} \rho_{i \downarrow} .
$$

Here, $\rho_{i \sigma}$ is the density operator (for $\sigma=\uparrow, \downarrow$ spins),

$$
\rho_{i \sigma}=\left(c_{i 1 \sigma}^{\dagger} c_{i 1 \sigma}+c_{i 2 \sigma}^{\dagger} c_{i 2 \sigma}-1\right)
$$

which counts the number of $\sigma$-spin fermions in both layers on site $i$ with respect to half filling. The repulsive $U>0$ (or attractive $U<0$ ) interaction drives spin $\langle\Sigma\rangle \neq 0$ (or charge $\langle\Delta\rangle \neq 0$ ) condensation, leading to a spin-density wave (SDW) [25] (or superconducting) phase that breaks the $U(1)_{\text {spin }}$ [or $U(1)_{\text {charge }}$ ] symmetry spontaneously. This process is illustrated in the schematic phase diagram Fig. 1(a).

\section{B. Easy-plane $J-Q$ model}

Our EPJQ model is a spin-1/2 system with anisotropic antiferromagnetic couplings, which we here define on the simple square lattice of $L^{2}$ sites and periodic boundary conditions. It is a "cousin" model of the previously studied $\mathrm{SU}(2)_{\text {spin }} J-Q_{3}$ model [54-56], which in turn is an extension of the original $J-Q$, or $J-Q_{2}$, model [28]. Starting from the spin-1/2 operator $\mathbf{S}_{i}$ on each site $i$, we define the singlet-projection operator on lattice link $i j$;

$$
P_{i j}=\frac{1}{4}-\mathbf{S}_{i} \cdot \mathbf{S}_{j}
$$

then the model Hamiltonian reads

$$
H_{J-Q}=-J \sum_{\langle i j\rangle}\left(P_{i j}+\Delta S_{i}^{z} S_{j}^{z}\right)-Q \sum_{\langle i j k l m n\rangle} P_{i j} P_{k l} P_{m n},
$$

where the $\Delta S_{i}^{z} S_{j}^{z}$ term for $\Delta \in(0,1]$ introduces the easyplane anisotropy that breaks the $\mathrm{SU}(2)_{\text {spin }}$ symmetry down to $U(1)_{\text {spin }}$ explicitly. In the $Q$ term, the index pairs $i j, k l$, and $m n$ correspond to links forming columns on $2 \times 3$ or $3 \times 2$ plaquettes, as illustrated in Fig. 1 of Ref. [54].

We study two cases of the anisotropy parameter $\Delta$ : the maximally planar case $\Delta=1$ and the less extreme case $\Delta=1 / 2$. In the latter case, we observe very good scaling behaviors indicating a continuous transition, with rapidly decaying (with the system size $L$ ) scaling corrections, while for $\Delta=1$ the behavior suggests a first-order transition. Thus, the model may harbor a tricritical point separating first-order and continuous transitions somewhere between $\Delta=1 / 2$ and $\Delta=1$. However, we leave the possible tricritical point to future investigation. As far as the duality 
is concerned, in this section we discuss our results for $\Delta=1 / 2$, and in Appendix B we present results for $\Delta=1$.

We set $J+Q=1$ in the simulations and define the control parameter as the ratio

$$
q=\frac{Q}{J+Q} .
$$

For small $q$, the model essentially reduces to an $X X Z$ model, which has an $X Y$ AFM ground state that breaks the $U(1)_{\text {spin }}$ symmetry spontaneously. When $q$ is large, the dimer interaction favors a VBS (columnar-dimerized) ground state, which breaks the lattice $C_{4}$ rotation symmetry as in the $\mathrm{SU}(2)_{\text {spin }} J-Q_{2}$ and $J-Q_{3}$ models $[28,32,53-$ 56], where previous QMC studies found a direct continuous AFM-VBS transition. Here, we demonstrate that the continuous transition persists in the EPJQ model, Eq. (11), with $\Delta=1 / 2$. The reason for choosing the $Q_{3}$ term (three-dimer interaction) instead of the simpler $Q_{2}$ interaction (two-dimer coupling) is that it produces a more robust VBS order when the ratio $q$ is large, thus leading to a smaller critical-point value [as in the SU(2) case [54,55] ] with more clearly observable flows to the VBS state on that side of the transition.

The $X Y$ AFM order in the EPJQ model can be directly probed by the local spin components $S^{x}$ and $S^{y}$, and we also study the critical fluctuations in the $S^{z}$ component. We often do not write out the staggered phase factor $(-1)^{x_{i}+y_{i}}$ corresponding to AFM order explicitly (here, $x_{i}$ and $y_{i}$ refer to the integer-valued lattice coordinates of site $i$ ); in fact, in the case of the $X Y$ anisotropy in a model with bipartite interactions, the phase can also simply be transformed away with a sublattice rotation (and then the $X Y$ AFM phase maps directly onto hard-core bosons in the superfluid state). In our simulations the staggered phase is absent for the $S^{x}$ and $S^{y}$ components but present for the $S^{z}$ component. The AFM-VBS transition is unstable towards an axial Zeeman field, when $H_{J-Q} \rightarrow H_{J-Q}+H_{Z}$, with

$$
H_{Z}=-h_{z} \sum_{i}(-1)^{x_{i}+y_{i}} S_{i}^{z}
$$

which drives the system to the spin-polarized phase with $\left\langle S^{z}\right\rangle \neq 0$, as illustrated in the schematic phase diagram in Fig. 1(b). Here we consider only $h=0$.

To study the columnar VBS (dimer) order realized in the EPJQ model, we define

$$
\begin{aligned}
& D_{i}^{x}=(-1)^{x_{i}} \mathbf{S}_{i} \cdot \mathbf{S}_{i+\hat{x}}, \\
& D_{i}^{y}=(-1)^{y_{i}} \mathbf{S}_{i} \cdot \mathbf{S}_{i+\hat{y}},
\end{aligned}
$$

where $i+\hat{x}$ and $i+\hat{y}$ denote neighbors of site $i$ in the positive $x$ and $y$ direction, respectively. At the critical point, the proposed self-duality (through the putative duality with
$N=2$ QED) implies that the $C_{4}$ rotation symmetry and the $U(1)_{\text {spin }}$ symmetry are enlarged into an emergent $O(4)$ symmetry, such that the components of the $O(4)$ vector (after some proper normalization),

$$
\mathbf{N}=\left(D^{x}, D^{y}, S^{x}, S^{y}\right),
$$

should all have the same scaling dimension [22].

\section{Duality relations}

Figure 1 summarizes the intuitive duality relations among the BH, EPJQ, and QED models; this can also be observed from the similarity of their four-quadrant phase diagrams [69]. To numerically prove the validity of these duality relations, in this work we investigate the following critical behaviors at the BSPT-Mott transition in the $\mathrm{BH}$ model:

$$
\begin{aligned}
\xi \sim\left|V-V_{c}\right|^{-\nu_{\mathrm{BH}}}, \\
\left\langle\rho_{i \uparrow} \rho_{i \downarrow} \rho_{j \uparrow} \rho_{j \downarrow}\right\rangle \sim\left|\mathbf{r}_{i j}\right|^{-1-\eta_{\mathrm{BH}}^{\rho}}, \\
\left\langle\Delta_{i}^{\dagger} \Delta_{j}\right\rangle \sim\left|\mathbf{r}_{i j}\right|^{-1-\eta_{\mathrm{BH}}^{\Delta}},
\end{aligned}
$$

where $\mathbf{r}_{i j}$ is the lattice vector separating the sites $i, j ; \xi$ is the correlation length of the critical $O(4)$ bosonic modes of the system; and the density $\rho_{i \sigma}$ and pairing $\Delta_{i}$ operators are defined in Sec. III A. We also study the following expected critical scaling behavior at the AFM-VBS transition in the EPJQ model:

$$
\begin{aligned}
\xi & \sim\left|Q-Q_{c}\right|^{-\nu_{J-Q}^{x y},} \\
\left\langle S_{i}^{z} S_{j}^{z}\right\rangle & \sim\left|\mathbf{r}_{i j}\right|^{-1-\eta_{J-Q}^{z},} \\
\left\langle S_{i}^{+} S_{j}^{-}\right\rangle & \sim\left|\mathbf{r}_{i j}\right|^{-1-\eta_{J-Q}^{x y},}
\end{aligned}
$$

where $\xi$ is the correlation length of the easy-plane spins.

If the duality in Eq. (1) is correct, and provided that $N=2$ QED is indeed the theory for the BSPT-Mott transition, then the exponents defined above must satisfy the following relationships [22]:

$$
\begin{aligned}
3-\frac{1}{\nu_{\mathrm{BH}}} & =\frac{1+\eta_{J-Q}^{z}}{2}, \\
3-\frac{1}{\nu_{J-Q}^{x y}} & =\frac{1+\eta_{\mathrm{QED}}}{2}=\frac{1+\eta_{\mathrm{BH}}^{\rho}}{2}, \\
\eta_{\mathrm{BH}}^{\Delta} & =\eta_{J-Q}^{x y} .
\end{aligned}
$$

Here, $\eta_{\mathrm{QED}}$ is the anomalous dimension of the fermion mass $\bar{\psi} \sigma^{3} \psi$, i.e., the $M$ mass term in our notation in Eq. (1a), which was numerically estimated in the recent lattice QED 
calculations in Ref. [34]. Equation (18) essentially means that the gauge-invariant operators that map to each other under the duality transformation must have the same scaling dimension at the critical point.

\section{RESULTS FOR THE EPJQ MODEL}

In this section, we present our numerical results at the continuous AFM-VBS phase transition of the EPJQ model, obtained using large-scale SSE-QMC [70,71] simulations. Here, we discuss only the case $\Delta=1 / 2$ in the Hamiltonian Eq. (11); some results for $\Delta=1$ are presented in Appendix B. In the SSE simulations we scale the inverse temperature as $\beta=2 L$, corresponding to the dynamic exponent $z=1\left(\beta \sim L^{z}\right)$ and staying in the regime where the system is close to its ground state for each $L$. We consider $L$ up to 44.

\section{A. Crossing-point analysis}

The first step is to determine the order of the transition and the position of the critical point (if the transition is continuous). To this end, following the recent example in Ref. [32] for the $\mathrm{SU}(2) J-Q$ model, we first analyze crossing points of finite-size Binder cumulants, defined for the AFM order parameter as

$$
U(q, L)=2-\frac{\left\langle M_{x y}^{4}\right\rangle}{\left\langle M_{x y}^{2}\right\rangle^{2}},
$$

where $M_{x y}^{2}$ is the square of the easy-plane magnetization operator,

$$
M_{x y}^{2}=\frac{1}{L^{4}} \sum_{i, j}(-1)^{i+j} S_{i}^{+} S_{j}^{-}
$$

and $M_{x y}^{4}$ is its square. The "phenomenological renormalization" underlying the crossing-point analysis and our technical implementations of it are discussed in Appendix A. Here, we show our numerical results and analyze them within the scaling relationships presented in Appendix A.

As shown in Fig. 2(a), curves of $U(q, L)$ graphed for different $L$ cross each other at points tending to a value $q_{c}$. In a finite-size system the deviation of $U(q, L)$ from the asymptotic crossing point depends on $L$ in a way that involves a scaling-correction exponent. For a finite-size pair $(L, 2 L)$, the crossing is at $\left[q_{c}^{*}(L), U_{c}^{*}(L)\right]$, and at a continuous transition one expects

$$
q_{c}^{*}(L)=q_{c}+a L^{-\left(1 / \nu_{J-Q}^{x y}+\omega\right)}
$$

where $\nu_{J-Q}^{x y}$ is the correlation-length exponent and $\omega$ is the smallest subleading exponent (which normally arises from the leading irrelevant field). As shown in Fig. 2(b), an
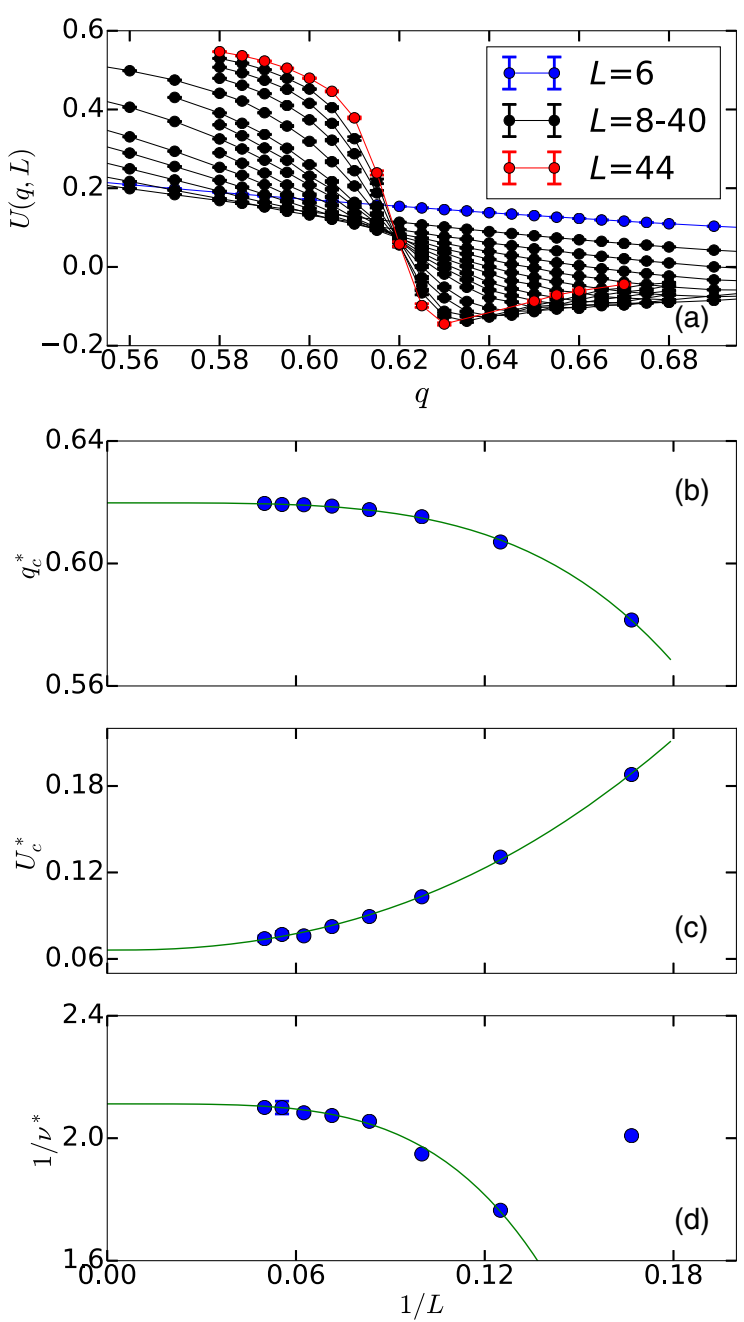

FIG. 2. Crossing-point analysis of the EPJQ model at $\Delta=1 / 2$. (a) $U(q, L)$ versus $q$ in the neighborhood of $q_{c}$ for several system sizes $L$. (b) The crossing points seen in (a) for system-size pairs $(L, 2 L)$, analyzed according to the expected finite-size scaling form, Eq. (21). The procedure including error analysis gives $q_{c}=$ $0.6197(2)$ and $1 / \nu_{J-Q}^{x y}+\omega=4.0(2)$. (c) A similar analysis of $U_{c}^{*}(L)$ based on Eq. (22), giving $\omega=2.3(1)$. (d) Finite-size estimates $\nu_{J-Q}^{x y, *}$ of the correlation-length exponent defined in Eq. (23), using the slopes of the cumulants at the $(L, 2 L)$ crossing points. Analysis according to Eq. (24) gives $\nu_{J-Q}^{x y}=0.48(2)$ for the exponent in the thermodynamic limit. In all fits, we exclude small system sizes until statistically sound fits are produced.

extrapolation with the above form to infinite size gives $q_{c}=0.6197(2)$ (where the number in parentheses indicates the one-standard-deviation error in the preceding digits, as obtained using numerical error propagation with normal-distributed noise added to the data points) and $1 / \nu_{J-Q}^{x y}+\omega=4.0(2)$. The finite-size crossing value of the cumulant itself, $U_{c}^{*}(L)$, should approach its thermodynamic limit $U_{c}$ as

$$
U_{c}^{*}(L)=U_{c}+a L^{-\omega} .
$$


This form is used in Fig. 2(b) and delivers $\omega=2.3(1)$. In principle, we can now extract $\nu_{J-Q}^{x y}$, though the so-obtained value and the independently determined value of $\omega$ in general should be viewed with some skepticism. The leading correction exponents, $\omega$ and $1 / \nu_{J-Q}^{x y}+\omega$ above, are often affected by neglected higher-order scaling corrections and should be regarded as "effective" exponents that flow to their correct values upon increasing the system sizes included (and excluding smaller sizes as needed to obtain statistically good fits). The quantities extrapolated to infinite size using the fits, which are what we are mainly interested in here, should not be seriously affected by potentially nonasymptotic correction forms, as discussed, e.g., in Ref. [32].

The correlation length exponent $\nu_{J-Q}^{x y}$ can be independently and more reliably obtained from the slope of the Binder cumulant as

$$
\frac{1}{\nu_{J-Q}^{x y, *}(L)}=\ln \left(\frac{U_{2}^{\prime}(2 L)}{U_{2}^{\prime}(L)}\right) \frac{1}{\ln (2)},
$$

where $U_{2}^{\prime}(L)$ is the derivative of $U(q, L)$ over $q$ evaluated at the crossing point between the $L$ and $2 L$ curves (which we extract by interpolating data close to the crossing point by cubic polynomials). The correlation-length exponent in the thermodynamic limit can be extracted from the expected leading finite-size form:

$$
\frac{1}{\nu_{J-Q}^{x y, *}(L)}=\frac{1}{\nu_{J-Q}^{x y}}+L^{-\omega}
$$

As shown in Fig. 2(d), the fit to this form is statistically good if the smallest system sizes are excluded, and an extrapolation then gives $\nu_{J-Q}^{x y}=0.48(2)$. Thus, the combination $\nu_{J-Q}^{x y}+\omega$ based on the independently evaluated two exponents is in remarkably good agreement with the value of the sum extracted directly using Eq. (21) with the data in Fig. 2(b). This serves as a good consistency check and indicates that the higher-order finite-size scaling corrections should be small (i.e., the following correction exponents beyond $\omega$ must either have much larger values or the prefactors must be small, or both). Further support for this scenario can be observed in Fig. 2(d), where the data point for the smallest system size shown has a very large deviation from the good fit to the other points, suggesting a very rapidly decaying correction.

In Fig. 2(a), one may worry about the fact that the Binder cumulant forms a minimum extending to negative values as the system size increases. A negative Binder cumulant often is taken as a sign of a first-order transition. However, it is now understood that also some continuous transitions are associated with a negative Binder cumulant in the neighborhood of the critical point, reflecting nonuniversal anomalies in the order-parameter distribution. Often the negative peak value grows slowly, e.g., logarithmically, with the system size, instead of the much faster volume proportionality expected at a first-order phase transition. This issue is discussed with examples from classical systems in Ref. [72]. Here, we do not see any evidence of a fast divergence of the peak value; thus, the transition should still be continuous.

\section{B. Anomalous dimensions}

To determine the anomlous dimensions, i.e., the critical correlation-function exponents $\eta_{J-Q}^{x y}$ and $\eta_{J-Q}^{z}$ in Eq. (17), we analyze the system-size dependence of the squares of the easy-plane off-diagonal spin order parameter $M_{x y}^{2}(L)$ in Eq. (20) and dimer order parameter

$$
D^{2}(L)=\frac{1}{2}\left[\left(D^{x}(L)\right)^{2}+\left(D^{y}(L)\right)^{2}\right],
$$

where the $x$ and $y$ dimer operators are the appropriate Fourier transforms of Eq.

$$
\begin{aligned}
& D^{x}=\frac{1}{L^{2}} \sum_{i}(-1)^{x_{i}} D_{i}^{x}, \\
& D^{y}=\frac{1}{L^{2}} \sum_{i}(-1)^{y_{i}} D_{i}^{y} .
\end{aligned}
$$

In the diagonal $S^{z}$ channel, we study the system-size dependence of the staggered magnetization:

$$
M_{z}^{2}=\frac{1}{L^{4}} \sum_{i, j}(-1)^{i+j} S_{i}^{z} S_{j}^{z}
$$

All these integrated correlation functions should scale as the correlation functions in Eq. (17) with the distance $\left|\mathbf{r}_{i j}\right|$ replaced by the system length $L$.

The dimer order parameter should be governed by the same exponent $\eta_{J-Q}^{x y}$ as the off-diagonal spin order parameter if the predicted $O(4)$ symmetry is manifested. In contrast, the diagonal magnetic order parameter is associated with a different (larger) anomalous dimension $\eta_{J-Q}^{z}$, according to the table in Fig. 1. We evaluate the order parameters at $q=0.620$, consistent with the value of $q_{c}$ determined in the previous section. Results are shown in Fig. 3 for system sizes up to $L=32$ and $L=40$ for the dimer and spin order parameters, respectively. In Figs. 3(a) and 3(b), we show that $M_{x y}^{2}$ and $D^{2}$ can be fitted with the same exponent, $\eta_{J-Q}^{x y}=0.13(3)$, while the fit to $M_{z}^{2}$ in Fig. 3(c) delivers a distinctively different exponent: $\eta_{J-Q}^{z}=0.91(3)$. 

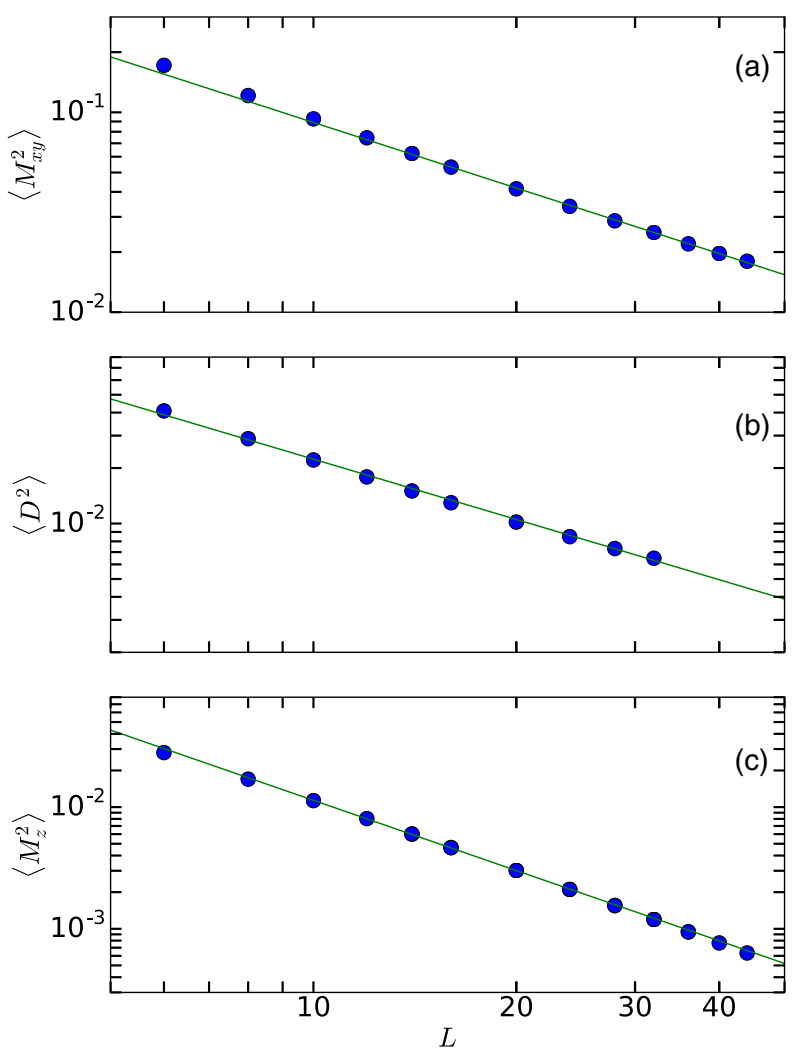

FIG. 3. Extraction of the anomalous dimensions $\eta_{J-Q}^{x y}$ and $\eta_{J-Q}^{z}$ of the EPJQ model at the estimated critical point $q_{c}$ for $\Delta=1 / 2$. The squares of order parameters are graphed versus $L$ and analyzed with power-law fits (lines on the log-log plots). The off-diagonal spin order parameter square $M_{x y}^{2}(L)$ in (a) and the dimer order parameter square $D^{2}(L)$ in (b) give $\eta_{J-Q}^{x y}=0.13(3)$. The diagonal spin order parameter square $M_{z}^{2}(L)$ in (c) gives $\eta_{J-Q}^{z}=0.91(3)$.

\section{RESULTS FOR THE BILAYER HONEYCOMB MODEL}

In this section, we present our numerical results on the continuous phase transition in the $\mathrm{BH}$ model, where an interaction-driven phase transition between a BSPT phase and a trivial Mott insulator is investigated via large-scale DQMC simulations $[24,64,73]$ in the ground-state projector version [74]. Acting with the operator $e^{-\Theta H}$ on a trial state (a Slater determinant) with the projection "time" $\Theta$ large enough for converging the finite system to its ground state, we simulate linear system sizes $L=12,15,18,21$, and 24 , with $\Theta=50$ for $L \leq 18, \Theta=55$ for $L=21$, and $\Theta=60$ for $L=24$. The imaginary-time discretization step is $\Delta \tau=0.05$, which is sufficiently small to not lead to any significant deviations of scaling behaviors from the $\Delta \tau=0$ limit.

\section{A. Continuous topological phase transition}

We first present simulation results supporting a continuous BSPT-Mott transition (as was also previously discussed
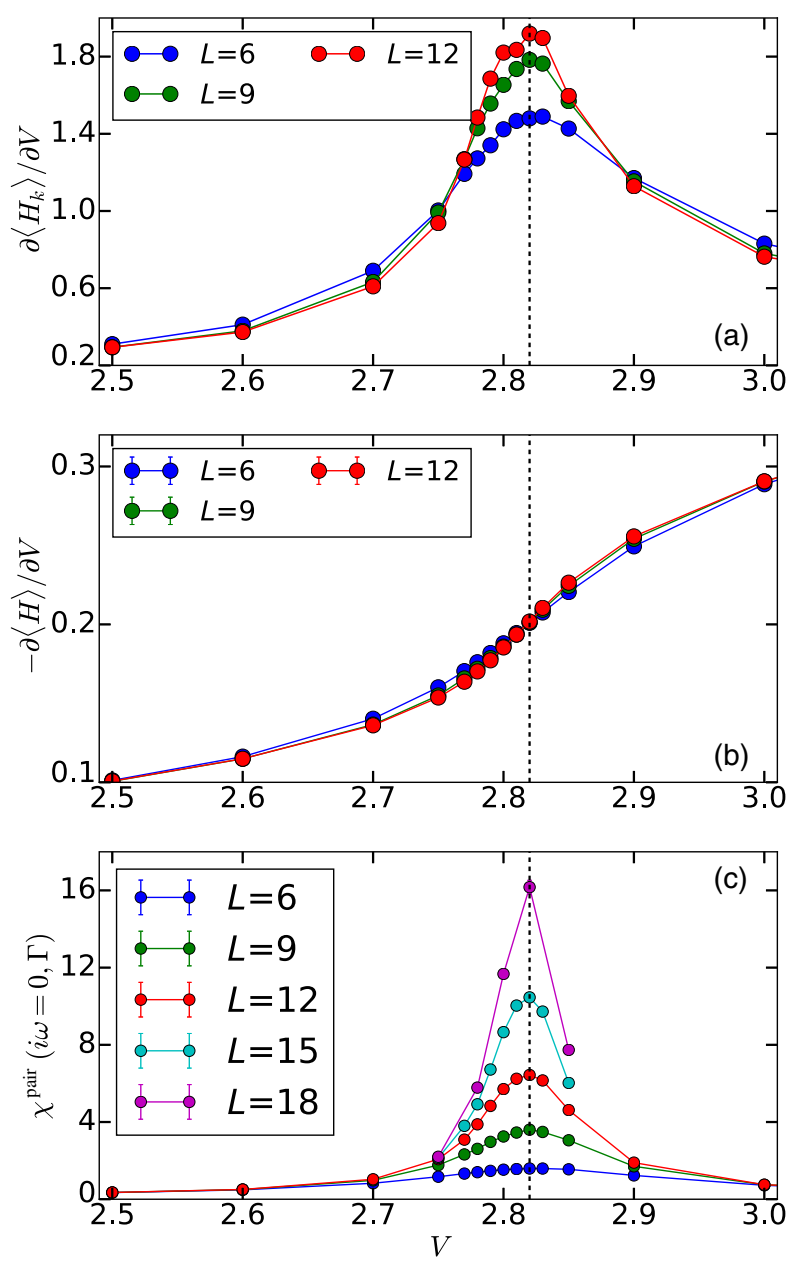

FIG. 4. DQMC results for the BH model close to its phase transition. The derivative with respect to the coupling $V$ of (a) the kinetic energy density and (b) the total ground-state energy density for linear system sizes $L=6,9$, and 12 . Panel (c) shows the zero-frequency susceptibility of the $O(4)$ vector in the pairing channel for $L=6,9,12,15,18$. The dashed line indicates our estimated critical point $V_{c}=2.82(1)$. In all cases the error bars are smaller than symbol size.

in Refs. [24,64]). Figure 4(a) shows the derivative of the kinetic energy density of the BH Hamiltonian Eq. (4) with respect to the control parameter $V$ of the phase transition,

$$
\frac{\partial\left\langle H_{k}\right\rangle}{\partial V}=-\frac{t}{N} \frac{\partial}{\partial V} \sum_{\langle i j\rangle}\left\langle c_{i}^{\dagger} c_{j}+\text { H.c. }\right\rangle \text {. }
$$

Here, a broad peak develops close to $V_{c}$, but there is no sign of a divergence, as would be expected at a first-order transition. Figure 4(b) shows the derivative of the groundstate energy density, which can be conveniently evaluated by invoking the Hellmann-Feynman theorem;

$\frac{\partial\langle H\rangle}{\partial V}=\left\langle\frac{\partial H}{\partial V}\right\rangle=\frac{1}{N} \sum_{i}\left\langle c_{i 1 \uparrow}^{\dagger} c_{i 2 \uparrow} c_{i 1 \downarrow}^{\dagger} c_{i 2 \downarrow}+\right.$ H.c. $\rangle$. 
For this derivative a first-order transition would lead to a sharp kink developing with increasing $L$ (corresponding to an avoided level crossing). The smooth behavior supports a continuous phase transition, though of course a very weak first-order transition would produce a visible singular behavior only for larger system sizes than we consider here.

To determine the phase-transition point $V_{c}$, we further calculate the zero-frequency susceptibility of the $O(4)$ vector (where we here take the on-site spin-singlet pairing operator), defined as

$$
\chi^{\mathrm{pair}}(i \omega=0, k=0)=\int_{0}^{+\infty} S(\tau, k=0) d \tau,
$$

where the dynamic pair-pair correlation function is defined as

$$
S(\tau, k=0)=\frac{1}{L^{2}} \sum_{i j} \frac{\left\langle\Delta_{i}^{\dagger}(\tau) \Delta_{j}+\Delta_{j}^{\dagger}(\tau) \Delta_{i}\right\rangle}{2},
$$

where $\Delta_{i}$ is defined in Eq. (7). As demonstrated in Fig. 4(c), this quantity exhibits a sharp peak, as expected at a gapless critical point with power-law correlations in both space and time. The divergence is considerably slower than the proportional to space-time-volume behavior expected at a first-order transition. Because of the large computational effort needed for these DQMC simulations, we do not have a sufficient density of points close to $V_{c}$ to carry out a systematic analysis of the drift of the peak position, but the data nevertheless allow us to roughly estimate the convergence to the critical point $V_{c} / t=2.82(1)$.

\section{B. $O(4)$ gap and $\mathrm{BH}$ correlation-length exponent}

We also extract the excitation gaps $\Delta_{\mathrm{O}(4)}$ corresponding to the $O(4)$ vectors defined in Eq. (7). According to Refs. $[24,64]$ and Eq. (31), the $O(4)$ gap is obtained from the imaginary-time decay of the dynamical $O(4)$ vector correlation function, and, as we discuss in Secs. II and III A, $O(4)$ bosonic modes are expected to become gapless (with power-law correlation) at the BSPT-Mott critical point. Results for $\Delta_{O(4)}$ as a function of $V / t$ for system sizes $L=6,9,12,15,18$ close to $V_{c}$ are presented in Fig. 5(a). As expected, $\Delta_{O(4)}$ from every system size $L$ exhibits a dip close to $V_{c}$, with the gap minimum decreasing with $L$, as expected with an emergent gapless point at $V_{c}$. In Fig. 5(b), we analyze the size dependence of the gap at three different coupling values: $V / t=2.80$, $2.82,2.85$. At $V / t=2.82, L \Delta_{O(4)}$ extrapolates linearly in $1 / L$ to a nonzero value, showing that the leading behavior of $\Delta_{O(4)}$ at $V_{c}$ is $1 / L$. This is in line with the expectation that the dynamic exponent $z=1$ at the BSPT-Mott transition (and is required also for the proposed duality). The behaviors of $\Delta_{O(4)} L$ at $V / t=2.80,2.85$ indicate eventual divergencies when $L \rightarrow \infty$, as expected on either side of the quantum-critical point. This constitutes strong evidence of a continuous transition, instead of a first-order transition at which one instead expects the avoided level-crossing gap to close exponentially fast with system size (instead of closing as $1 / L^{z}$ at a continuous transition).

To extract the correlation-length exponent $\nu_{\mathrm{BH}}$, we perform data collapse with the $O(4)$ gap away from the critical point, as shown in Fig. 5(c). Here, we focus on the regime $V>V_{c}$, where we find less scaling corrections than for $V<V_{c}$ and an almost perfect data collapse according to the expected quantum-critical form,

$$
\Delta_{O(4)} L=f\left[\left(V / V_{c}-1\right) L^{1 / \nu_{\mathrm{BH}}}\right]
$$
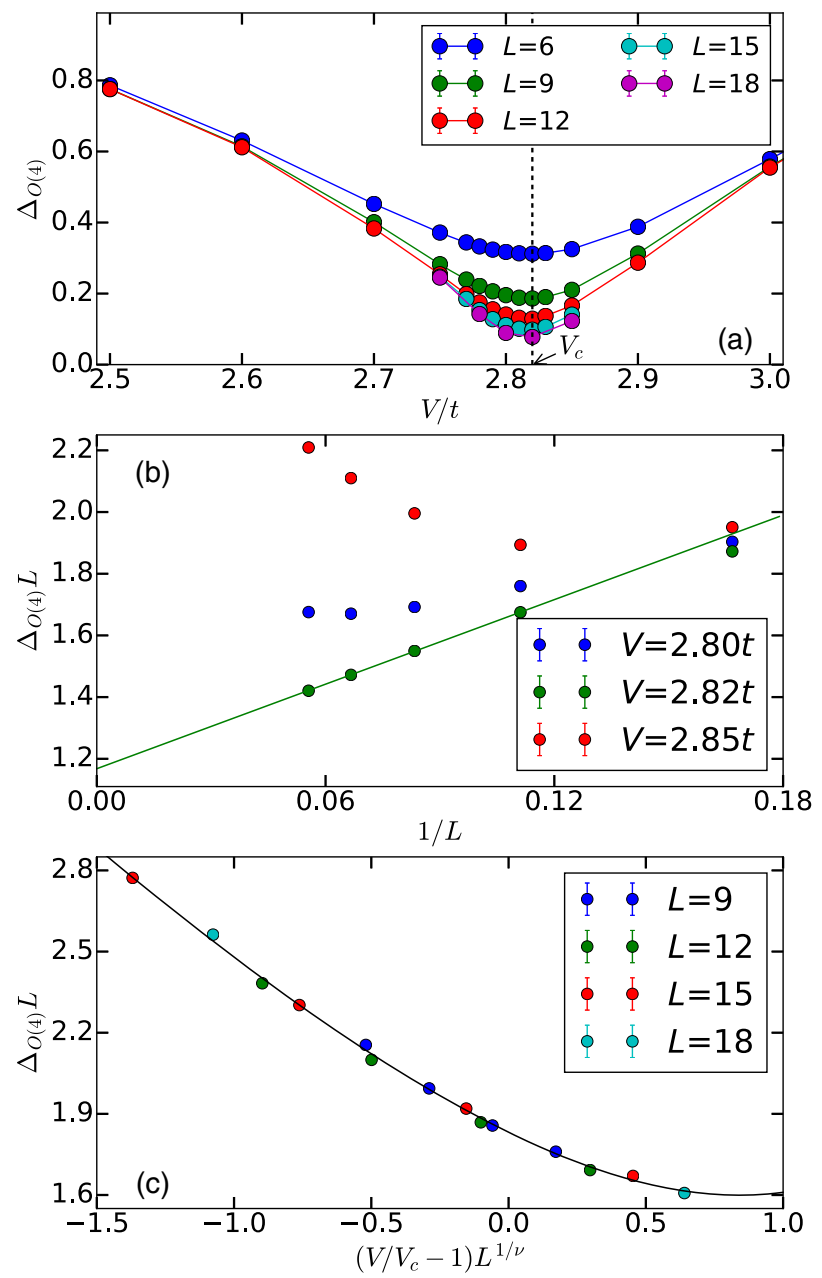

FIG. 5. (a) Excitation gaps computed from the imaginary-time decay of the $O(4)$ vector across the BSPT-Mott transition for the BH model with $L=6,9,12,15,18$. A gap closing at $V_{c}=2.82 t$ (vertical dashed line) is expected. (b) Finite-size scaling of $L \Delta_{O(4)}$ for $V / t=2.80,2.82,2.85$ versus the inverse system size. The behavior at $V / t=2.82$ indicates $z=1$. (c) Data collapse of the data in (a) according to the expected scaling form Eq. (32), yielding $\nu_{\mathrm{BH}}=0.53(5)$ and $V_{c} / t=2.80(1)$. 
Treating both $\nu_{\mathrm{BH}}$ and $V_{c}$ as free parameters, the best data collapse delivers $\nu_{\mathrm{BH}}=0.53(5)$ and $V_{c} / t=2.80(1)$. This value of $V_{c}$ agrees quite well with the result $V_{c}=2.82(1)$ estimated roughly from the susceptibility peak in Fig. 4, adding further credence to the analysis of the critical point even with the rather limited range of system sizes accessible (as compared with the EPJQ model). Moreover, since we already determined $\eta_{J-Q}^{z}=0.91(3)$ in the EPJQ model, we can use the duality relationship in Eq. (18a) to predict that the correlation-length exponent of the $\mathrm{BH}$ model should be $\nu_{\mathrm{BH}} \sim 0.49(2)$, which is fully consistent with the number determined from the $O(4)$ gap.

\section{Anomalous dimensions}

Finally, we study the critical equal-time correlations in the $\mathrm{BH}$ model. Here, we use $V=2.817$ for the longest simulations. This value is within the error bars of the critical value $V_{c}=2.82(1)$ and, as we also show, there are no statistically detectable differences between data at $V=$ 2.820 and 2.817 for the quantities we study in this section.

Using one of the components of the $O(4)$ order parameter, $\left\langle\Delta_{i}^{\dagger} \Delta_{j}\right\rangle$ with $\Delta_{i}$ defined in Eq. (7), we again construct a squared order parameter. We can use the susceptibility Eq. (31) to define a corresponding equal-time spatially integrated correlation function,

$$
\frac{S_{\Delta}}{L^{2}}=\frac{S(\tau=0, k=0)}{L^{2}},
$$

where the normalization gives the same scaling behaviors as in Eq. (16) with the distance $\left|\mathbf{r}_{i j}\right|$ replaced by $L$. The analysis illustrated in Fig. 6(a) indicates a very good powerlaw scaling, with deviations seen only for the smallest system size (which we exclude from the fit). The fit delivers the exponent $\eta_{\mathrm{BH}}^{\Delta}=0.10(1)$, which is fully consistent with the EPJQ exponent $\eta_{J-Q}^{X Y}=0.13(3)$ obtained in Sec. IV B. Hence, the duality relation Eq. (18c) is satisfied to within the statistical errors.

In principle, the anomalous dimension can also be obtained from the susceptibility in Fig. 5(c). Standard scaling arguments give that the peak height of a generic susceptibility $\chi$ should scale as $\chi_{\text {peak }} \propto L^{2-\eta}$ (when the dynamic exponent $z=1$ ). We find that the peak in $\chi^{\text {pair }}$ scales approximately as $L^{2}$, i.e., $\eta^{\Delta}$ is very small, but here there appears to be significant scaling corrections. Moreover, there is large variation of the values for the largest size, $L=18$, close to the critical point, and we would need additional points to reliably estimate the peak value. We therefore cannot obtain an independent meaningful estimate for $\eta_{\mathrm{BH}}^{\Delta}$ from these data.

We next test the duality relation Eq. (18b). With $\nu_{J-Q}^{x y}=$ $0.48(3)$ obtained in Sec. IV A, we expect $\eta_{\mathrm{BH}}^{\rho} \approx 1$. Further, according to Ref. [34], the exponent $\eta_{\mathrm{BH}}^{\rho}$ should be equal to the anomalous mass dimension $\eta_{\mathrm{QED}}$ of $N=2 \mathrm{QED}$, for
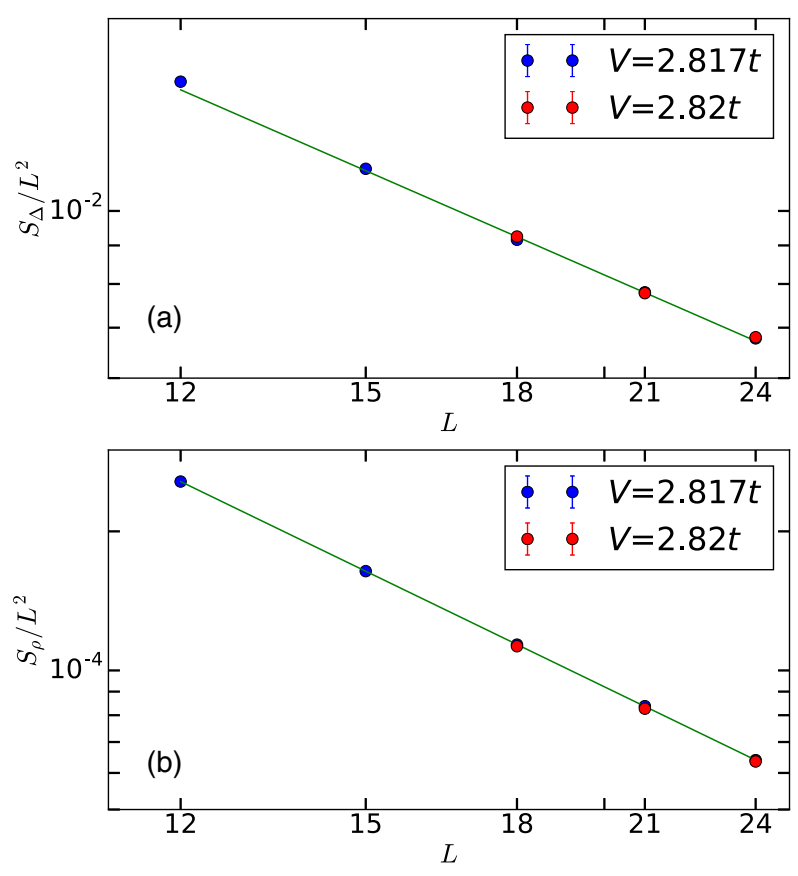

FIG. 6. Analysis of the anomalous dimensions $\eta_{\mathrm{BH}}^{\Delta}$ and $\eta_{\mathrm{BH}}^{\rho}$ of the $\mathrm{BH}$ model from squared order parameters close to the critical point. Only the $V=2.817$ data points for each quantity are used in the fits, and the three points for $V=2.82$ do not exhibit any deviations from the $V=2.817$ values within the error bars. Panel (a) shows the $O(4)$ order parameter defined in Eq. (33). A powerlaw fit (straight line on the $\log$-log scale) to the $L \geq 15$ data delivers the exponent $\eta_{\mathrm{BH}}^{\Delta}=0.10(1)$. (b) The pair-density order parameter Eq. (34). Here the power-law fit works well for all system sizes and we obtain $\eta_{\mathrm{BH}}^{\rho}=1.00(1)$.

which the value $\eta_{\mathrm{QED}}=1.0(2)$ has been obtained from Monte Carlo simulations [34]. Thus, we already have good consistency following from the predicted duality between $\eta_{\mathrm{QED}}$ and $\nu_{J-Q}^{x y}$. Turning to the more direct test with the $\mathrm{BH}$ model, in Fig. 6(b) we plot the squared order parameter corresponding to the pair density,

$$
\frac{S_{\rho}}{L^{2}}=\frac{1}{L^{4}} \sum_{i, j}\left\langle\rho_{i \uparrow} \rho_{i \downarrow} \rho_{j \uparrow} \rho_{j \downarrow}\right\rangle .
$$

For this quantity, as shown in Fig. 6(b), all five system sizes available give results fully consistent with a power-law decay, with no statistically visible scaling corrections. The fit to the five data points gives the anomalous dimension $\eta_{\mathrm{BH}}^{\rho}=1.00(1)$, which is consistent with both $\eta_{\mathrm{QED}}$ and $\nu_{J-Q}^{x y}$, but with a significantly smaller statistical error. Thus, the duality relation in Eq. (18b) is also confirmed to within error bars.

It is remarkable that the $\mathrm{BH}$ model actually seems to give better results (smaller error bars) for the anomalous dimensions than the EPJQ model, even though system lengths roughly twice as large are used for the latter. The 
reason is that the statistical errors on the raw data are smaller in the DQMC simulations. It is still possible that there are scaling corrections present that are not clearly visible with such a small range of system sizes, and there may then be some corrections to the exponents beyond the purely statistical error bars (1 standard deviation) reported above. The EPJQ results are important in this regard as they seem to show no significant corrections even with the considerably larger range of system sizes. The lack of significant corrections is also supported by the good agreement between the nontrivial BH and EPJQ exponents, as predicted by the duality conjecture.

\section{DISCUSSION}

We perform detailed numerical tests on the recently proposed duality summarized by the predicted exponent relationships between the BH and EPJQ models in Eq. (18). The relationships are confirmed within statistical precision at the level of a few percent in the values of the critical exponents. The thus confirmed duality of the underlying low-energy quantum field theories, Eq. (1), is of great importance and interest in condensed-matter physics, because it relates two seemingly different quantum phase transitions that have been individually under intense studies during the past several years: the bosonic topological phase transition and the easy-plane deconfined quantum phase transition. The duality is derived using the more basic dualities between field theories that involve only one flavor of matter field, and sometimes also a Chern-Simons term of the dynamical gauge field.

As a consequence of confirming the particular relationship between critical exponents, our study also provides quantitative evidence for the underlying basic dualities for theories with one flavor of matter field [40-45]. These basic dualities supported by our work represent a significant step in our understanding of $(2+1)$ D conformal field theories. They also form the foundation of a large number of other recently proposed dualities [20,48,50,52,75-79]. Moreover, they lend support to many other dualities that follow from the same logic and reasoning, such as the duality of Majorana fermions discussed in Refs. [80,81].

To follow up on our results and insights presented here, additional numerical investigations are called for to check other predictions made within these proposed dualities. For example, in Ref. [22] it was proposed that the Gross-Neveu fixed point of the $N=2$ QED is dual to the $\mathrm{SU}(2)$ version of the $\mathrm{NCCP}^{1}$ model, and also has an emergent $\mathrm{SO}(5)$ symmetry. This symmetry has recently been discussed within $\mathrm{SU}(2)$ deconfined quantum criticality as well, and quite convincing results pointing in this direction were seen in a three-dimensional loop model [62]. Scaling with the same anomalous dimension for both spin and dimer correlators had also been observed already some time ago in the $\mathrm{SU}(2) J-Q$ model [82]. Although we identify the $N=2$ QED as the bosonic topological phase transition in our bilayer honeycomb lattice model, we are thus far unable to find the corresponding Gross-Neveu fixed point. Identifying the additional interactions that will be required to drive this transition in the $\mathrm{BH}$ model is an important topic for further research.

Following previous computational studies of deconfined quantum phase transitions with $\mathrm{SU}(2)$ spin-rotation symmetry in $J-Q$ models $[28,32,54]$, we here identify a lattice model-the EPJQ model-hosting a continuous phase transition between the U(1) (planar) Néel and VBS states. The fact that this phase transition is continuous is in itself an important discovery, given that $U(1)$ deconfined quantum criticality had essentially been declared nonexistent, due to unexplained hints of first-order transitions in some other planar models and what seems like definite proofs in other cases [35,37,38,83]. Here (as further discussed in Appendix B), we show that the EPJQ model defined in Eq. (11) can host first-order or continuous transitions, depending on the degree of spin anisotropy parametrized by the Ising coupling $\Delta$ in Eq. (11). Thus, there should be a tricritical point separating first-order and continuous transitions at some value of $\Delta$. In the recent Ref. [38], it was argued that a tricritical point only exists above a large threshold value of the number of spin components $N$, and numerical results were interpreted as showing that $N$ as large as $\approx 20$ was required before any continuous transitions appear. Our results obtained here explain this apparent disagreement by the degree of anisotropy. The spin model considered in Ref. [38] is always strongly anisotropic (as is the case also for other planar models considered previously), while the EPJQ model has tunable anisotropy and we demonstrate that continuous transitions obtain only up to moderate values of the anisotropy. Thus, the critical value of $N$ at which a tricritical point appears extends down to $N=2$, and the boson-fermion duality holds for the continuous transitions.

At $\Delta=1 / 2$, we find scaling behaviors with apparently much less influence of scaling corrections than in the SU(2) $J-Q$ model at its deconfined critical point [32]; i.e., the leading correction exponent $\omega$ is much larger in the EPJQ model. Interestingly, in both cases the correlation-length exponent is unusually small, close to $1 / 2$, while wellstudied transitions such as the $O(N)$ transitions in three dimensions have exponents close to $2 / 3$. Given its small scaling corrections and likely tricritical point between $\Delta=$ $1 / 2$ and $\Delta=1$, the EPJQ model opens doors for future detailed studies on exotic phase transitions beyond the Landau paradigm.

An interesting question following from our work is whether the duality of the theories in Eq. (1) could also be extended to the tricritical point that we argue for within the EPJQ model. The corresponding tricritical point had been previously identified field theoretically within the $\mathrm{CP}^{N-1}$ model, but only for a very large number $N$ of spin components [38]. Our work implies that it should exist also 
in the $\mathrm{CP}^{1}$ model for weak planar anisotropy, and one may then ask whether a corresponding tricritical point should exist in the fermionic theory, and whether it will be dual to the one in the $\mathrm{CP}^{1}$ model. We plan to consider this both theoretically and numerically in future work.

\section{ACKNOWLEDGMENTS}

The authors thank Chong Wang and Meng Cheng for helpful discussions. Y.Q. Q., Y,-Y.H., Z.-Y.L., and Z. Y.M. are supported by the Ministry of Science and Technology of China under Grant No. 2016YFA0300502, the National Science Foundation of China under Grants No. 91421304, No. 11421092, No. 11474356, No. 11574359, and No. 11674370, and the National Thousand-Young-Talents Program of China. Y. Q. Q. would like to thank Boston University for support under its Condensed Matter Theory Visitors Program. Y.-Y.H. acknowledges the Cultivation of Outstanding Innovative Talents Program 2016 of Renmin University of China. The work of A. S. is partly supported through the Partner Group program between the Indian Association for the Cultivation of Science (Kolkata) and the Max Planck Institute for the Physics of Complex Systems (Dresden). A. W. S. is supported by the NSF under Grant No. DMR-1410126 and would also like to thank the Institute of Physics, Chinese Academy of Sciences for visitor support. C. X. is supported by the David and Lucile Packard Foundation and NSF Grant No. DMR-1151208. We thank the following institutions for allocation of CPU time: the Center for Quantum Simulation Sciences in the Institute of Physics, Chinese Academy of Sciences, the Physical Laboratory of High Performance Computing in the Renmin University of China, the Tianhe-1A platform at the National Supercomputer Center in Tianjin, and the Tianhe-2 platform at the National Supercomputer Center in Guangzhou.

\section{APPENDIX A: CROSSING-POINT ANALYSIS}

To determine the critical point and the critical exponents in an unbiased manner, we adopt the crossing-point analysis applied and tested for 2D Ising and $\mathrm{SU}(2) J-Q$ models in Ref. [32]. Such analysis can be further traced back to Fisher's "phenomenological renormalization," which was first numerically tested with transfer-matrix results for the Ising model in Ref. [84]. Reference [32] presented systematic procedures for a statistically sound application of these techniques with Monte Carlo data. For easy reference we here summarize how we adapt the method to the EPJQ model we study in this paper. For the $\mathrm{BH}$ model, due to the much larger computational cost of the DQMC simulations, we do not have data for enough system sizes to carry out the analysis in this way, and we instead apply other scaling methods in Sec. V.

Considering a generic critical point, with $\delta=q-q_{c}$ defined as the distance to the critical point. For example, $q$ can be the control parameter $q=Q /(J+Q)$ that we use for the EPJQ model or it could be $T-T_{c}$ for a finite-temperature transition. For any observable $O$, the standard finite-size scaling form is

$$
O(\delta, L)=L^{-\kappa / \nu} f\left(\delta L^{1 / \nu}, \lambda L^{-\omega}\right),
$$

where we, for the sake of simplicity, consider only one irrelevant field $\lambda$ and the corresponding subleading exponent $\omega$. At the critical point, one can Taylor expand the scaling function:

$$
O(\delta, L)=L^{-\kappa / \nu}\left(a_{0}+a_{1} \delta L^{1 / \nu}+b_{1} L^{-\omega}+\cdots\right) .
$$

If one now takes two system sizes, e.g., $L_{1}=L$ and $L_{2}=r L$ $(r>1)$, and traces the crossing points $\delta^{*}(L)$ of curves $O\left(\delta, L_{1}\right)$ and $O\left(\delta, L_{2}\right)$ versus $\delta$, one finds

$$
\begin{aligned}
\delta^{*}(L)= & \frac{a_{0}}{a_{1}} \frac{1-r^{-\kappa / \nu}}{r^{(1-\kappa) / \nu-1}} L^{-1 / \nu} \\
& +\frac{b_{1}}{a_{1}} \frac{\left.1-r^{-(\kappa / \nu+\omega}\right)}{r^{(1-\kappa) / \nu-1}} L^{-(1 / \nu+\omega)} .
\end{aligned}
$$

Now, if the quantity $O$ is asymptotically size independent (dimensionless) at the critical point, for example, the Binder cumulant (which we write here with a constant and factor corresponding to a planar vector order parameter),

$$
U=2\left(1-\frac{\left\langle m_{x y}^{4}\right\rangle}{2\left\langle m_{x y}^{2}\right\rangle^{2}}\right),
$$

then the corresponding exponent $\kappa=0$, the first term in Eq. (A3) with $O=U$ vanishes, and we obtain the following form for the size-dependent crossing point $\delta^{*}(L)$ :

$$
\delta^{*}(L)=q_{c}^{*}(L)-q_{c} \propto L^{-(1 / \nu+\omega)}
$$

hence, the shift of the finite-size critical point $q_{c}^{*}(L)$ is approaching the asymptotic value $q_{c}$ as $L^{-(1 / \nu+\omega)}$.

In practice, one can interpolate within a set of points for each system size by a fitted polynomial, e.g., of cubic or quadratic order, and then use these polynomials to find the crossing points. This is illustrated in Fig. 7. Error bars can be obtained by repeating the fits multiple times to data with Gaussian-distributed noise added. The scaling behavior of $q_{c}$ predicted by Eq. (A5) can be clearly seen in Fig. 2(b), from which the result $1 / \nu+\omega=4.0(2)$ for the EPJQ model is obtained.

In addition to obtaining $q_{c}^{*}$ and the exponent combination $1 / \nu+\omega$ from the crossing points of the cumulant (or, in principle, some other dimensionless quantity), one can also use the value $U_{c}^{*}$ of the quantity at $q_{c}^{*}$, as well as the derivatives at $q_{c}^{*}$, to acquire $\nu$ and $\omega$ independently. We next discuss the derivations underlying these forms. 


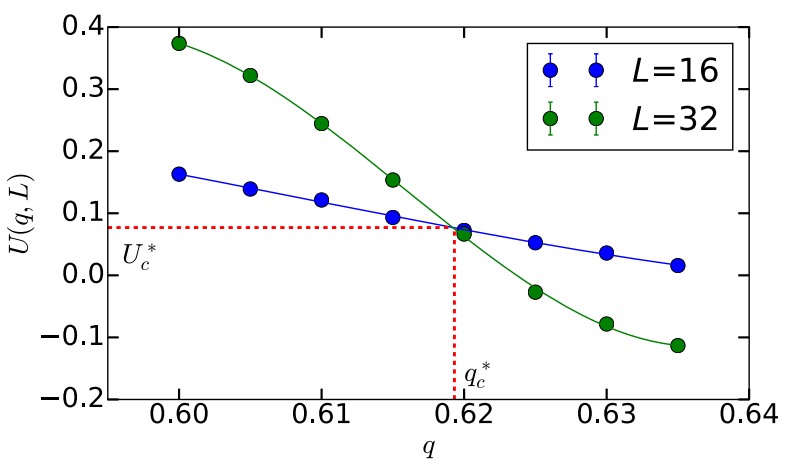

FIG. 7. Example of finite-size crossing points $q_{c}^{*}(L), U_{c}^{*}(L)$ of the Binder cumulant, here for the EPJQ model with $L=16$ and $L=32$. The data are here fitted to third-order polynomials. The crossing point and derivatives at the crossing point can be determined from the fitted polynomials.

By inserting $\delta^{*}(L)$ into Eq. (A2), one can obtain the value of the observable at the finite-size critical point (or, more precisely, the critical point depending on the two sizes, $L$ and $r L) q_{c}^{*}(L)$. It scales as

$$
O^{*}(L)=L^{-\kappa / \nu}\left(a+b L^{-\omega}+\cdots\right) .
$$

Again, for a dimensionless quantity $(\kappa=0)$ such as $U$, the deviation of the value at the crossing point from the value in the thermodynamic limit vanishes with increasing size according to $U_{c}^{*}(L)-U_{c} \propto L^{-\omega}$, an example of which can be seen in Fig. 2(c) - in this case, the power-law fit gives the value $\omega=2.3(1)$ of the subleading exponent.

The last step of the analysis of the single dimensionless quantity is to determine $\nu$ in an independent manner. To this end, one can expand the quantity $U$ (or any other dimensionless quantity) close to the critical point,

$U(\delta, L)=a_{0}+a_{1} \delta L^{1 / \nu}+b_{1} L^{-\omega}+c_{1} \delta L^{1 / \nu-\omega}+\cdots$,

and take the derivative $U^{\prime}(\delta, L)$ with respect to $\delta$ (in practice, with respect to $q)$ :

$$
U^{\prime}(\delta, L)=a_{1} L^{1 / \nu}+c_{1} L^{1 / \nu-\omega}+\cdots .
$$

Again, we take two system sizes, $L_{1}=L$ and $L_{2}=r L$, and at the crossing point $\delta^{*}(L)$ of the two curves for these system sizes one has

$$
\begin{aligned}
U^{\prime}\left(\delta^{*}, L\right) & =a_{1} L^{1 / \nu}+c_{1} L^{1 / \nu-\omega}, \\
U^{\prime}\left(\delta^{*}, r L\right) & =a_{1}(r L)^{1 / \nu}+c_{1}(r L)^{1 / \nu-\omega} .
\end{aligned}
$$

Here, we can take the difference of the logarithms of the two equations and obtain

$$
\ln \left(\frac{U^{\prime}\left(\delta^{*}, r L\right)}{U^{\prime}\left(\delta^{*}, L\right)}\right)=\frac{1}{\nu} \ln (r)+d L^{-\omega}+\cdots,
$$

or, in other words, one can define a finite-size estimate of the correlation-length exponent $\nu^{*}(L)$ from the finite-size crossing point as

$$
\frac{1}{\nu^{*}(L)}=\frac{1}{\ln (r)} \ln \left(\frac{U^{\prime}\left(\delta^{*}, r L\right)}{U^{\prime}(\delta, L)}\right)
$$

It can be seen that Eq. (A11) approaches the thermodynamic limit correlation-length exponent $\nu$ at the rate $1 / \nu^{*}(L)-1 / \nu=g L^{-\omega}+\cdots$. This behavior is seen nicely in Fig. 2(d), where the extrapolation to infinite size gives $\nu_{J-Q}^{x y}=0.48(2)$.

In principle, one can also combine the above method for a dimensionless quantity with some other quantity $A$, e.g., an order parameter or a long-distance correlation function. Interpolating the data for two system sizes, $A(L)$ and $A(r L)$ at the crossing point of the dimensionless quantity, one can take the logarithm of the ratio and analyze it in a manner similar to the slope estimate of $1 / \nu$, to yield a series of finite-size estimates for the power law governing the size dependence of $A$. This method circumvents the need to know the critical-point value exactly. In practice, since $q_{c}$ converges fast, it is also appropriate to just use this value and analyze the size dependence of the quantity $A$ at this fixed coupling value $q=q_{c}$, as we do in the main text.

\section{APPENDIX B: FULLY PLANAR EPJQ MODEL}

In the main text, we discuss the $X Y$ AFM-VBS transition at a fixed anisotropy parameter $\Delta=1 / 2$ of the EPJQ model. We here provide some more information on the dependence on $\Delta$.

In the extreme planar limit $\Delta \rightarrow 1$ of Eq. (11), we have no $S^{z} S^{z} J$ term and the Hamiltonian is

$$
H_{J-Q}=J \sum_{\langle i j\rangle}\left(S_{i}^{x} S_{j}^{x}+S_{i}^{y} S_{j}^{y}\right)+Q \sum_{\langle i j k l m n\rangle} D_{i j} D_{k l} D_{m n} .
$$

We analyze SSE-QMC results for this model in the same way as we do for $\Delta=1 / 2$ in the main text, using a crossing-point analysis. The results of this analysis show a distinctively different behavior for $\Delta=1$, pointing to a first-order transition in this case. Results for the $L$ dependent quantities based on the $X Y$ Binder cumulant are shown in Fig. 8. As an aside, we mention here that the offdiagonal spin correlations can be measured as diagonal ones upon performing a basis rotation with the $z$ spin components transformed into the $x$ components, and vice versa. Simulating the system with SSE-QMC in this rotated basis speeds up the simulations, and we did this for $\Delta=1$. We therefore have results for larger systems in this case than for the case $\Delta=1 / 2$ reported in the main text.

It is clear from Fig. 8 that we can obtain a good estimate of the critical point, but the cumulant itself and the correlation-length exponent do not exhibit the clear-cut power-law corrections of the type that we see for the 

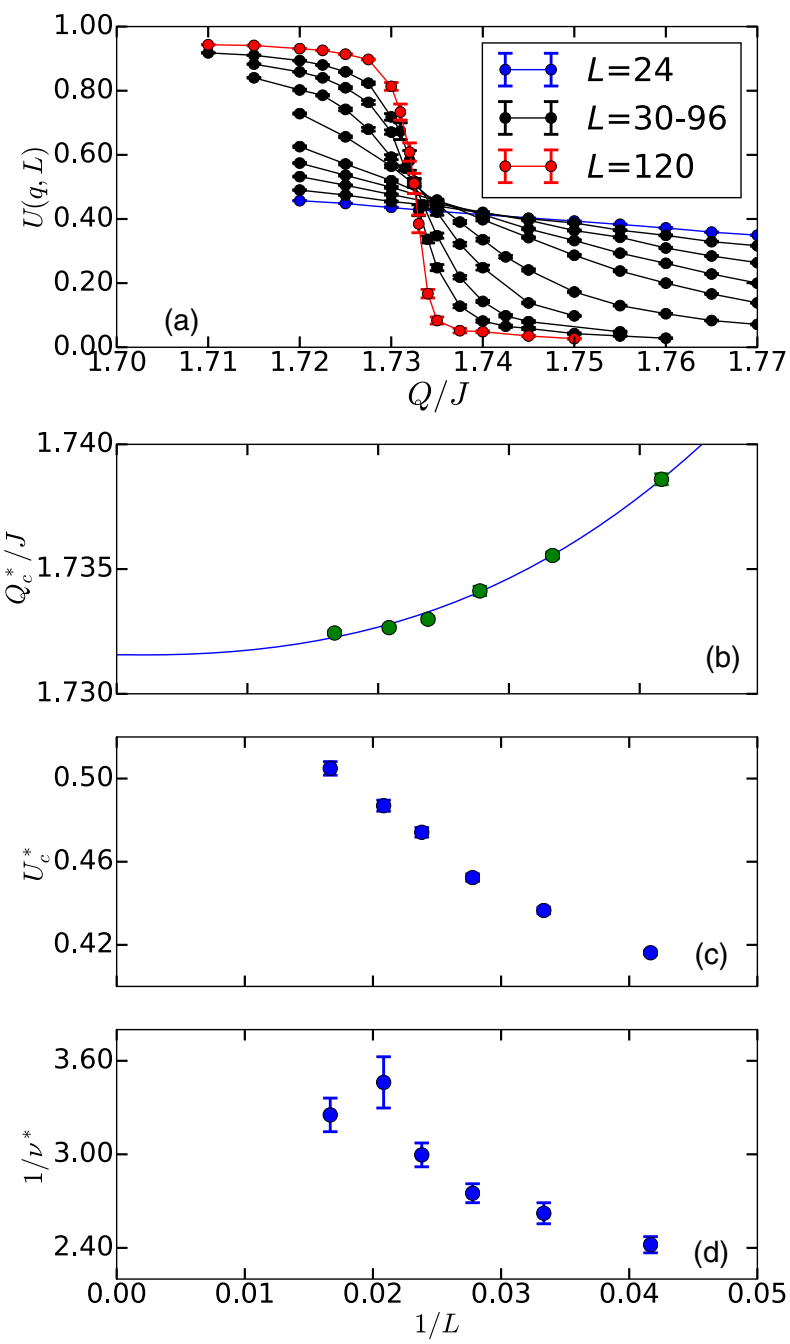

FIG. 8. Crossing-point analysis of the extreme, $\Delta=1$, version of the EPJQ model, Eq. (B1). (a) The magnetic Binder cumulant versus the coupling $Q$ in the neighborhood of the phase transition. (b) Size dependence of the critical point defined as the crossing point of $U$ versus $Q$ for system sizes $L$ and $2 L$, with a power-law fit giving $Q_{c}=1.732(2)$. (c) The cumulant evaluated at the crossing points. Here, it is not clear whether the asymptotic behavior has been reached, and we refrain from carrying out a fit. (d) The finite-size correlation length. Here, as well, we do not present any fit, as the asymptotic behavior is not yet clear.

$\Delta=1 / 2$ model in Sec. IV. The fact that $1 / \nu^{*}$ is larger than 3 suggests that the transition may actually be of first order in this case. If so, we would expect the values to eventually tend exactly to 3 , and the data are consistent with this behavior.

If the transition indeed is of first order, the order parameter should be nonzero at the transition point, reflecting coexistence of the magnetic and nonmagnetic phases. Indeed, as shown in Fig. 9(a), an extrapolation using a trivial $1 / L$ correction, as expected asymptotically for a $2 \mathrm{D}$ system breaking a continuous symmetry, indicates
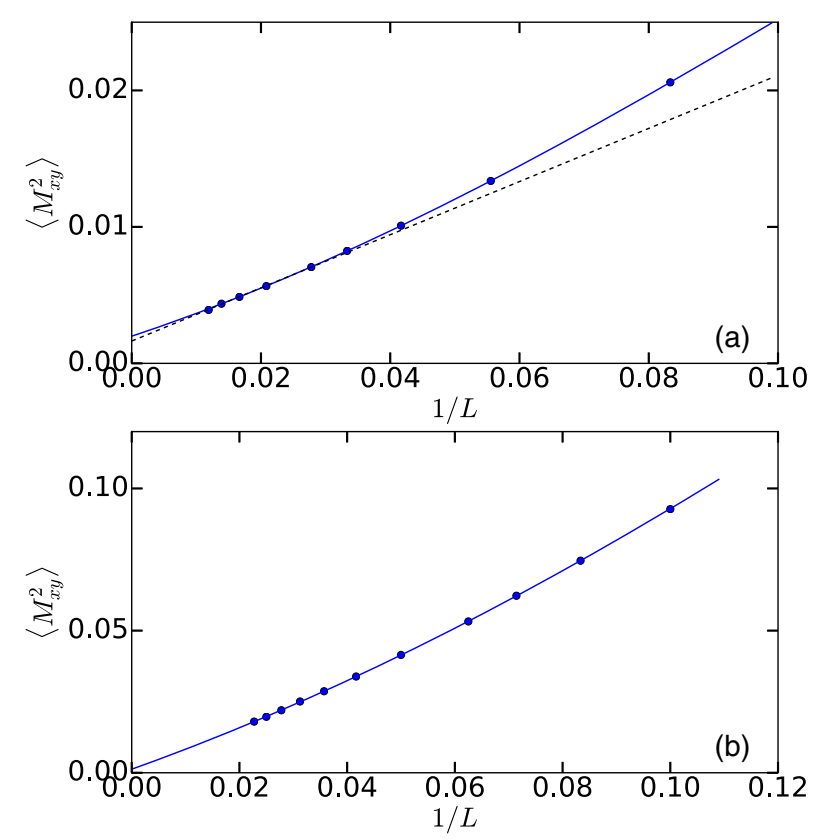

FIG. 9. Comparison of the $X Y$ magnetization squared of the $\Delta=1 / 2$ and $\Delta=1$ EPJQ models at their respective critical points. In (a), fits indicating a non-vanishing order parameter in the thermodynamic limit are indicated, with the dashed line corresponding to a purely linear correction and the solid curve to a quadratic form. In (b), a second-order fit is shown. The extrapolated value at $L=\infty$ is 0 to within two error bars.

a clearly nonzero value in the thermodynamic limit. The extrapolated value changes only slightly if a higherorder $\left(1 / L^{2}\right)$ correction is added (also expected if the order parameter is nonvanishing). In contrast, as shown in Fig. 9(b), the data for the $\Delta=1 / 2$ model are fully consistent with no magnetization in the thermodynamic limit. Here, the polynomial fit is strictly not correct, since a nontrivial power is expected at the critical point (which we confirm in the main text), but the extrapolation nevertheless indicates consistency with a vanishing order parameter at the transition in this case.

These results strongly suggest that there is a tricritical point separating a continuous and first-order transition in the EPJQ model somewhere between $\Delta=1 / 2$ and $\Delta=1$, which we plan to investigate further in a future study.

[1] H. A. Kramers and G. H. Wannier, Statistics of the TwoDimensional Ferromagnet. Part I, Phys. Rev. 60, 252 (1941).

[2] J. B. Kogut, An Introduction to Lattice Gauge Theory and Spin Systems, Rev. Mod. Phys. 51, 659 (1979).

[3] R. Savit, Duality in Field Theory and Statistical Systems, Rev. Mod. Phys. 52, 453 (1980).

[4] M. E. Peskin, Mandelstam-'t Hooft Duality in Abelian Lattice Models, Ann. Phys. (N.Y.) 113, 122 (1978). 
[5] C. Dasgupta and B. I. Halperin, Phase Transition in a Lattice Model of Superconductivity, Phys. Rev. Lett. 47, 1556 (1981).

[6] M. P. A. Fisher and D. H. Lee, Correspondence between Two-Dimensional Bosons and a Bulk Superconductor in a Magnetic Field, Phys. Rev. B 39, 2756 (1989).

[7] Here, the term "noncompact" means that the $U(1)$ gauge flux is a conserved quantity.

[8] C. Montonen and D. Olive, Magnetic Monopoles as Gauge Particles?, Phys. Lett. B 72, 117 (1977).

[9] N. Seiberg, Electric-Magnetic Duality in Supersymmetric Non-Abelian Gauge Theories, Nucl. Phys. B435, 129 (1995).

[10] N. Seiberg and E. Witten, Electric-Magnetic Duality, Monopole Condensation, and Confinement in $n=2$ Supersymmetric Yang-Mills Theory, Nucl. Phys. B426, 19 (1994).

[11] N. Seiberg and E. Witten, Monopoles, Duality and Chiral Symmetry Breaking in $n=2$ Supersymmetric $Q C D$, Nucl. Phys. B431, 484 (1994).

[12] J. L. Cardy and E. Rabinovici, Phase Structure of $Z_{p}$ Models in the Presence of a Parameter, Nucl. Phys. B205, 1 (1982).

[13] J. L. Cardy, Duality and the Parameter in Abelian Lattice Models, Nucl. Phys. B205, 17 (1982).

[14] E. Witten, On S-Duality in Abelian Gauge Theory, Sel. Math. Sov. 1, 383 (1995).

[15] S. Coleman, Quantum Sine-Gordon Equation as the Massive Thirring Model, Phys. Rev. D 11, 2088 (1975).

[16] S. Mandelstam, Soliton Operators for the Quantized Sine-Gordon Equation, Phys. Rev. D 11, 3026 (1975).

[17] A. Luther and I. Peschel, Single-Particle States, Kohn Anomaly, and Pairing Fluctuations in One Dimension, Phys. Rev. B 9, 2911 (1974).

[18] E. Witten, Non-Abelian Bosonization in Two Dimensions, Commun. Math. Phys. 92, 455 (1984).

[19] T. Giamarchi, Quantum Physics in One Dimension, International Series of Monographs (Clarendon Press, Oxford, 2004).

[20] A. Karch and D. Tong, Particle-Vortex Duality from 3D Bosonization, Phys. Rev. X 6, 031043 (2016).

[21] A. C. Potter, C. Wang, M. A. Metlitski, and A. Vishwanath, Realizing Topological Surface States in a LowerDimensional Flat Band, arXiv:1609.08618.

[22] C. Wang, A. Nahum, M. A. Metlitski, C. Xu, and T. Senthil, Deconfined Quantum Critical Points: Symmetries and Dualities, Phys. Rev. X 7, 031051 (2017).

[23] K. Slagle, Y.-Z. You, and C. Xu, Exotic Quantum Phase Transitions of Strongly Interacting Topological Insulators, Phys. Rev. B 91, 115121 (2015).

[24] Y.-Y. He, H.-Q. Wu, Y.-Z. You, C. Xu, Z. Y. Meng, and Z.-Y. Lu, Bona fide Interaction-Driven Topological Phase Transition in Correlated Symmetry-Protected Topological States, Phys. Rev. B 93, 115150 (2016).

[25] H.-Q. Wu, Y.-Y. He, Y.-Z. You, T. Yoshida, N. Kawakami, C. Xu, Z. Y. Meng, and Z.-Y. Lu, Visualizing a Bosonic Symmetry Protected Topological Phase in an Interacting Fermion Model, Phys. Rev. B 94, 165121 (2016).
[26] T. Grover and A. Vishwanath, Quantum Phase Transition between Integer Quantum Hall States of Bosons, Phys. Rev. B 87, 045129 (2013).

[27] Y.-M. Lu and D.-H. Lee, Quantum Phase Transitions between Bosonic Symmetry-Protected Topological Phases in Two Dimensions: Emergent $\mathrm{QED}_{3}$ and Anyon Superfluid, Phys. Rev. B 89, 195143 (2014).

[28] A. W. Sandvik, Evidence for Deconfined Quantum Criticality in a Two-Dimensional Heisenberg Model with FourSpin Interactions, Phys. Rev. Lett. 98, 227202 (2007).

[29] T. Senthil, A. Vishwanath, L. Balents, S. Sachdev, and M. P. A. Fisher, Deconfined Quantum Critical Points, Science 303, 1490 (2004).

[30] T. Senthil, L. Balents, S. Sachdev, A. Vishwanath, and M.P. A. Fisher, Quantum Criticality beyond the LandauGinzburg-Wilson Paradigm, Phys. Rev. B 70, 144407 (2004).

[31] T. Senthil and M. P. A. Fisher, Competing Orders, Nonlinear Sigma Models, and Topological Terms in Quantum Magnets, Phys. Rev. B 74, 064405 (2006).

[32] H. Shao, W. Guo, and A. W. Sandvik, Quantum Criticality with Two Length Scales, Science 352, 213 (2016).

[33] T. Grover and A. Vishwanath, Quantum Phase Transition between Integer Quantum Hall States of Bosons, Phys. Rev. B 87, 045129 (2013).

[34] N. Karthik and R. Narayanan, Scale Invariance of ParityInvariant Three-Dimensional QED, Phys. Rev. D 94, 065026 (2016).

[35] A. B. Kuklov, M. Matsumoto, N. V. Prokof'ev, B. V. Svistunov, and M. Troyer, Deconfined Criticality: Generic First-Order Transition in the SU(2) Symmetry Case, Phys. Rev. Lett. 101, 050405 (2008).

[36] S. D. Geraedts and O. I. Motrunich, Monte Carlo Study of a $U(1) \times U(1)$ System with $\pi$-Statistical Interaction, Phys. Rev. B 85, 045114 (2012).

[37] J. D'Emidio and R. K. Kaul, First-Order Superfluid to Valence-Bond Solid Phase Transitions in Easy-Plane $\mathrm{SU}(n)$ Magnets for Small n, Phys. Rev. B 93, 054406 (2016).

[38] J. D'Emidio and R. K. Kaul, New Easy-Plane $\mathbb{C}^{N-1}$ Fixed Points, Phys. Rev. Lett. 118, 187202 (2017).

[39] W. Chen, M. P. A. Fisher, and Y.-S. Wu, Mott Transition in an Anyon Gas, Phys. Rev. B 48, 13749 (1993).

[40] N. Seiberg, T. Senthil, C. Wang, and E. Witten, A Duality Web in Dimensions and Condensed Matter Physics, Ann. Phys. (Amsterdam) 374, 395 (2016).

[41] D. T. Son, Is the Composite Fermion a Dirac Particle?, Phys. Rev. X 5, 031027 (2015).

[42] C. Wang and T. Senthil, Time-Reversal Symmetric U(1) Quantum Spin Liquids, Phys. Rev. X 6, 011034 (2016).

[43] C. Wang and T. Senthil, Dual Dirac Liquid on the Surface of the Electron Topological Insulator, Phys. Rev. X 5, 041031 (2015).

[44] C. Wang and T. Senthil, Half-Filled Landau Level, Topological Insulator Surfaces, and Three-Dimensional Quantum Spin Liquids, Phys. Rev. B 93, 085110 (2016).

[45] M. A. Metlitski and A. Vishwanath, Particle-Vortex Duality of Two-Dimensional Dirac Fermion from Electric-Magnetic 
Duality of Three-Dimensional Topological Insulators, Phys. Rev. B 93, 245151 (2016).

[46] S. Kachru, M. Mulligan, G. Torroba, and H. Wang, Nonsupersymmetric Dualities from Mirror Symmetry, Phys. Rev. Lett. 118, 011602 (2017).

[47] S. Kachru, M. Mulligan, G. Torroba, and H. Wang, Bosonization and Mirror Symmetry, Phys. Rev. D 94, 085009 (2016).

[48] D. F. Mross, J. Alicea, and O. I. Motrunich, Explicit Derivation of Duality between a Free Dirac Cone and Quantum Electrodynamics in $(2+1)$ Dimensions, Phys. Rev. Lett. 117, 016802 (2016).

[49] A more precise form of the dual $(2+1) \mathrm{D}$ QED is given in Ref. [40].

[50] C. Xu and Y.-Z. You, Self-Dual Quantum Electrodynamics as Boundary State of the Three-Dimensional Bosonic Topological Insulator, Phys. Rev. B 92, 220416 (2015).

[51] O. I. Motrunich and A. Vishwanath, Emergent Photons and Transitions in the O(3) Sigma Model with Hedgehog Suppression, Phys. Rev. B 70, 075104 (2004).

[52] P.-S. Hsin and N. Seiberg, Level/Rank Duality and ChernSimons-Matter Theories, J. High Energy Phys. 09 (2016) 095.

[53] A. W. Sandvik, S. Daul, R. R. P. Singh, and D. J. Scalapino, Striped Phase in a Quantum xy Model with Ring Exchange, Phys. Rev. Lett. 89, 247201 (2002).

[54] J. Lou, A. W. Sandvik, and N. Kawashima, Antiferromagnetic to Valence-Bond-Solid Transitions in Two-Dimensional $\mathrm{SU}(n)$ Heisenberg Models with Multispin Interactions, Phys. Rev. B 80, 180414 (2009).

[55] A. Sen and A. W. Sandvik, Example of a First-Order Néel to Valence-Bond-Solid Transition in Two Dimensions, Phys. Rev. B 82, 174428 (2010).

[56] A. W. Sandvik, Continuous Quantum Phase Transition between an Antiferromagnet and a Valence-Bond Solid in Two Dimensions: Evidence for Logarithmic Corrections to Scaling, Phys. Rev. Lett. 104, 177201 (2010).

[57] A. Nahum, J. T. Chalker, P. Serna, M. Ortuño, and A. M. Somoza, 3D Loop Models and the $\mathrm{CP}^{n-1}$ Sigma Model, Phys. Rev. Lett. 107, 110601 (2011).

[58] K. Harada, T. Suzuki, T. Okubo, H. Matsuo, J. Lou, H. Watanabe, S. Todo, and N. Kawashima, Possibility of Deconfined Criticality in $S U(N)$ Heisenberg Models at Small N, Phys. Rev. B 88, 220408 (2013).

[59] S. Pujari, K. Damle, and F. Alet, Néel-State to ValenceBond-Solid Transition on the Honeycomb Lattice: Evidence for Deconfined Criticality, Phys. Rev. Lett. 111, 087203 (2013).

[60] S. Pujari, F. Alet, and K. Damle, Transitions to ValenceBond Solid Order in a Honeycomb Lattice Antiferromagnet, Phys. Rev. B 91, 104411 (2015).

[61] A. Nahum, J. T. Chalker, P. Serna, M. Ortuño, and A. M. Somoza, Deconfined Quantum Criticality, Scaling Violations, and Classical Loop Models, Phys. Rev. X 5, 041048 (2015).

[62] A. Nahum, P. Serna, J. T. Chalker, M. Ortuño, and A. M. Somoza, Emergent SO(5) Symmetry at the Néel to ValenceBond-Solid Transition, Phys. Rev. Lett. 115, 267203 (2015).
[63] X.-F. Zhang, Y.-C. He, S. Eggert, R. Moessner, and F. Pollmann, Continuous Easy-Plane Deconfined Phase Transition on the Kagome Lattice, arXiv:1706.05414.

[64] Y.-Y. He, H.-Q. Wu, Z. Y. Meng, and Z.-Y. Lu, Topological Invariants for Interacting Topological Insulators. II. Breakdown of Single-Particle Green's Function Formalism, Phys. Rev. B 93, 195164 (2016).

[65] Z. Y. Meng, T. C. Lang, S Wessel, F. F. Assaad, and A. Muramatsu, Quantum Spin Liquid Emerging in TwoDimensional Correlated Dirac Fermions, Nature (London) 464, 847 (2010).

[66] Z. Y. Meng, H.-H. Hung, and T. C. Lang, The Characterization of Topological Properties in Quantum Monte Carlo Simulations of the Kane-Mele-Hubbard Model, Mod. Phys. Lett. B 28, 1430001 (2014).

[67] C. L. Kane and E. J. Mele, Quantum Spin Hall Effect in Graphene, Phys. Rev. Lett. 95, 226801 (2005).

[68] Y.-Z. You, Z. Bi, D. Mao, and C. Xu, Quantum Phase Transitions between Bosonic Symmetry-Protected Topological States without Sign Problem: Nonlinear Sigma Model with a Topological Term, Phys. Rev. B 93, 125101 (2016).

[69] Y. You and Y.-Z. You, Stripe Melting and a Transition between Weak and Strong Symmetry Protected Topological Phases, Phys. Rev. B 93, 195141 (2016).

[70] A.W. Sandvik, Stochastic Series Expansion Method with Operator-Loop Update, Phys. Rev. B 59, R14157 (1999).

[71] O.F. Syljuåsen and A. W. Sandvik, Quantum Monte Carlo with Directed Loops, Phys. Rev. E 66, 046701 (2002).

[72] S. Jin, A. Sen, and A. W. Sandvik, Ashkin-Teller Criticality and Pseudo-First-Order Behavior in a Frustrated Ising Model on the Square Lattice, Phys. Rev. Lett. 108, 045702 (2012).

[73] Y.-Y. He, H.-Q. Wu, Z. Y. Meng, and Z.-Y. Lu, Topological Invariants for Interacting Topological Insulators. I. Efficient Numerical Evaluation Scheme and Implementations, Phys. Rev. B 93, 195163 (2016).

[74] F. F. Assaad and H. G. Evertz, in Computational ManyParticle Physics, Lecture Notes in Physics, Vol. 739, edited by H. Fehske, R. Schneider, and A. Weiße (Springer, Berlin, 2008), pp. 277-356.

[75] M. Cheng and C. Xu, Series of $(2+1)$-Dimensional Stable Self-Dual Interacting Conformal Field Theories, Phys. Rev. B 94, 214415 (2016).

[76] A. Karch, B. Robinson, and D. Tong, More Abelian Dualities in $2+1$ Dimensions, J. High Energy Phys. 01 (2017) 017.

[77] F. Benini, P.-S. Hsin, and N. Seiberg, Comments on Global Symmetries, Anomalies, and Duality in $(2+1) \mathrm{D}$, J. High Energy Phys. 04 (2017) 135.

[78] W.-H. Hsiao and D. T. Son, Duality and Universal Transport in Mixed-Dimension Electrodynamics, Phys. Rev. B 96, 075127 (2017).

[79] D. F. Mross, J. Alicea, and O. I. Motrunich, Symmetry and Duality in Bosonization of Two-Dimensional Dirac Fermions, arXiv:1705.01106 [Phys. Rev. X (to be published)]. 
[80] M. A. Metlitski, A. Vishwanath, and C. Xu, Duality and Bosonization of $(2+1)$-Dimensional Majorana Fermions, Phys. Rev. B 95, 205137 (2017).

[81] O. Aharony, F. Benini, P.-S. Hsin, and N. Seiberg, ChernSimons-Matter Dualities with SO and USp Gauge Groups, J. High Energy Phys. 02 (2017) 072.

[82] A. W. Sandvik, Finite-Size Scaling and Boundary Effects in Two-Dimensional Valence-Bond Solids, Phys. Rev. B 85, 134407 (2012).
[83] S. D. Geraedts and O. I. Motrunich, Lattice Realization of a Bosonic Integer Quantum Hall State: Trivial Insulator Transition and Relation to the Self-Dual Line in the Easy-Plane NCCP1 Model, arXiv:1705.06308 [Phys. Rev. B (to be published)].

[84] J. M. Luck, Corrections to Finite-Size-Scaling Laws and Convergence of Transfer-Matrix Methods, Phys. Rev. B 31, 3069 (1985). 